\title{
Fuzzy Logic Based Coverage and Cost Effective Placement of Serving Nodes for 4G and Beyond Cellular Networks
}

\author{
Arthi Murugadass ${ }^{1}$ and Arulmozhivarman Pachiyappan ${ }^{2}$ \\ ${ }^{1}$ School of Electronics Engineering, VIT University, Vellore, Tamil Nadu, India \\ ${ }^{2}$ School of Electrical Engineering, VIT University, Vellore, Tamil Nadu, India \\ Correspondence should be addressed to Arthi Murugadass; arthimdas@gmail.com
}

Received 14 July 2016; Revised 13 October 2016; Accepted 23 October 2016; Published 16 January 2017

Academic Editor: Giovanni Pau

Copyright (c) 2017 Arthi Murugadass and Arulmozhivarman Pachiyappan. This is an open access article distributed under the Creative Commons Attribution License, which permits unrestricted use, distribution, and reproduction in any medium, provided the original work is properly cited.

\begin{abstract}
The densification of serving nodes is one of the potential solutions to maximize the spectral efficiency per unit area. This is preposterous on account of conventional base stations (BS) for which site procurement is costly. Long term evolution-advanced (LTE-A) defines the idea of heterogeneous networks (HetNets), where BSs with different coverage and capacity are utilized to guarantee the quality of service $(\mathrm{QoS})$ requirements of the clients. To maximize the transmission quality of the clients in the coverage holes, LTE-A also defines multihop relay (MHR) networks, where the relay stations (RSs) are also placed along with the BSs. Unfortunately, the placement approaches for HetNet and MHR serving nodes are not standardized. In this work, two different approaches like site selection with maximum service coverage (SSMSC) and site selection with minimum placement cost (SSMPC) are proposed, which identifies the required number of serving nodes, their types, and the placement locations to maximize the coverage and to maintain the placement cost (PC) within the limits of the total placement budget. The simulation results demonstrate that the proposed approaches are computationally less complex and offer enhanced performance in terms of aggregate PC, coverage, and power proportion compared to the other conventional approaches.
\end{abstract}

\section{Introduction}

The factors like path loss, shadowing, environmental noise, and interference between different cells not only degrade the quality of the transmitted signal but also reduce the service coverage range significantly [1]. Apart from the voice communication, the service providers should also support the large bandwidth demanding multimedia data services like video streaming, high definition online television, online games, and teleconference to the service requesting users. The network capacity has to be maximized to support the clients in the dead areas and in the hotspots [2]. LTE-A proposed various aspects like massive multiple-input multiple-output (MIMO) and carrier aggregation to maximize the network capacity by improving the link spectral efficiency [3, 4]. The link spectral efficiency performance of the $3 \mathrm{G}$ and $4 \mathrm{G}$ systems is almost closer to the theoretical bounds specified in standards. Thus, any effort to maximize the network capacity by improving the link spectral efficiency is meaningless. In $4 \mathrm{G}$ and beyond standards, the network capacity is maximized by increasing the density of the serving nodes [5].

The placement of more number of evolved universal mobile telecommunications system (UMTS) terrestrial radio access network (E-UTRAN) NodeBs (eNB) or BSs in the given geographic area is extremely costly. This also increases the intercell interference and degrades the spectrum allocation. The idea of HetNet is introduced in LTE-A to improve the transmitted signal quality and to extend the service coverage, while not increasing the network cost. LTE-A defines four different types of eNBs, namely, macro, micro, pico, and femto $[4,6]$. All these eNBs coexist in the same geographic area. Macro, micro, and pico eNBs are installed by the network operators and femto eNB is installed by the customers.

LTE and IEEE 802.16e are developed and promoted for high rate packet services [1]. Due to the path loss and attenuation, the user equipment (UE) located near the cell boundary experiences poor data rate and QoS [7]. The 
placement of small eNBs is inefficient, where the wired backhaul connections are unavailable and expensive. Apart from HetNet, LTE-A and IEEE $802.16 \mathrm{j}$ standards proposed the idea of MHR networks, where the RSs are placed along with the eNBs to maximize the coverage and capacity [8]. Particularly fixed RSs are placed in the dead areas, where there always exist a serious signal attenuation.

The cost and physical size of small eNBs and RSs are much lower than macro eNB [9]. The introduction of small eNBs and RSs may reduce the distance between the communicating nodes. This minimizes the path loss and increases the transmission signal quality. The small eNBs and RSs increase the spectral efficiency per unit area. Unfortunately, the placement of small eNBs and RSs is not mentioned in any of the standards. The improper placements may lead to larger transmission delay, PC, and interference. This also reduces the transmission quality and service coverage.

The conventional $2 \mathrm{G}$ and $3 \mathrm{G}$ standards use macrocell deployment (MCD) approach, where all the BSs have the same coverage range [4]. To support cell edge clients with high quality connection, macro eNB needs to increase the transmitted power level. This increases the total power consumption. This approach always places macro eNBs irrespective of the number of UEs. This increases the intereNB interference and PC.

$\mathrm{Ge}$ et al. proposed a random deployment approach (RAND) for placing different types of RSs on the given geographic area [10]. But the population distribution and the traffic demands are not considered in this approach. Tsourakis and Voudouris proposed a static average weighting approach (SWA) for RS deployment [11]. Here RSs are placed based on the radio signal strength indicator (RSSI) and carrier to noise and interference ratio constraints. The throughput performance of both the constraints is compared under line of sight (LOS) and non-line of sight (NLOS) conditions. Shin et al. proposed a dynamic weighting approach (DWA) for RS placement based on the constraints like hop count, rate of the link, and rate of the shared link [12]. This approach dynamically redeploys the RSs when there is a change in population distribution and the abovementioned constraints. But the dynamic redeployment may increase the computational complexity. Fu et al. proposed an RS placement approach for Manhattan-like environment [13]. This approach places different types of RS to guarantee the following two features: There should be a LOS connection from all the RSs to the serving eNB and a NLOS connection from a RS to all other interfering RSs. The approaches proposed in [13-15] aim to maximize the system throughput by selecting the RSs which can support high data rate.

In literature, only a few works discuss the placement of eNB and RS together. Yu et al. proposed an eNB and RS placement approach for IEEE 802.16 j using a cost function [14]. This approach discards various performance metrics like throughput, coverage, cost, and power consumption. Kim et al. proposed a cost-effective RS placement approach for coverage extension [15]. This approach determines the optimal number of eNBs and RSs based on the placement budget and traffic requirements. To validate the optimal performance, this approach is tested under three different environments like metropolitan, suburban, and rural.

Chang et al. proposed an adaptive cost based RS deployment (ACRD) approach by considering five different quality factors like transmission quality, PC, service coverage with and without population, and RS overlapping index [1]. Then a cost function is modeled in terms of all the abovementioned quality factors. The optimization problem is solved for the least network cost. This approach also considers the placement of three different types of RS, namely, fixed, nomadic, and mobile. The simulation results demonstrate that ACRD approach maximizes the transmission quality and service coverage and minimizes the system cost and inter-RS interference compared to the RAND [10], SWA [11], and DWA [12] approaches. It is also shown that ACRD approach places more number of RSs on the high density populated areas than the other areas. But this approach is tested for a small $2 \mathrm{~km} \times 2 \mathrm{~km}$ geographic area with smaller number of UEs. This approach considers free space propagation model which is unrealistic. This approach also assumes maximum number of RS placement combinations. The computational complexity of this approach increases with the number of RS placement combinations. In all the above approaches, the effect of link overloading and the handoff performance are not considered.

$\mathrm{Lu}$ and Liao proposed an integer linear programming model based joint BS and RS placement (JBRP) approach for IEEE $802.16 \mathrm{j}$ [16]. JBRP approach is proposed to satisfy both coverage and budget constraints. Through numerical results, it is proved that the proposed approach maximizes the system throughput while not maximizing the PC. This approach suffers by the traffic load imbalance issue. This may increase the packet queuing delay. It is also assumed that the RS candidate sites are the same as the locations of demand nodes (DNs) and BS candidate sites are the corners of DNs. These assumptions are unrealizable and outlandish. Chang and Lin proposed a uniform clustering based eNB and RS placement approach for IEEE $802.16 \mathrm{j}$ networks [9]. This approach utilizes the modified $K$-means algorithm to form macro eNB clusters based on the UE locations. This approach functions in two phases, namely, eNB-RS selection and eNB-RS placement. This approach outperforms JBRP approach in terms of coverage and budget requirements. In this approach, there is no special consideration to improve the system throughput. Chang and Lin (2015) proposed a fuzzy logic based eNB placement approach, where the coverage ratio (CR) and traffic ratio (TR) of each candidate site are given as the input for fuzzy inference engine (FIE) [17]. The selection factor (SF) corresponding to each candidate site is the output from the FIE. The authors have framed the Mamdani triangular membership functions for CR, TR, and SF. Based on two input parameters, they have framed nine rules. The proposed approach chooses the candidate sites with highest SF based on the coverage and budget constraints. It is also shown that the candidate sites identified using the fuzzy logic offers significant performance gain over the other conventional approaches. But, the authors failed to consider inter-eNB and inter-RS interferences, which may degrade the performance of the proposed approach. The authors also 
considered the simple free space propagation model, which is impractical. The authors also failed to consider realistic path loss models, shadowing effects, and environmental noise. This approach utilizes fuzzy logic for eNB placement and not for RS placement. This approach also fails to consider the small cells like micro and pico. The performance of the proposed approach under link traffic overloading is also not discussed.

Ting et al. proposed a multiobjective genetic algorithm based HetNet transmitter placement approach for worldwide interoperability for microwave access (WiMAX) and wireless fidelity (WiFi) networks [18]. This approach maximizes the service coverage while minimizing the PC. This approach first decides the number of HetNet transmitters required to meet the expected objectives and then decides their heterogeneity. The numerical results prove that the approach proposed in [18] outperforms various single objective HetNet placement approaches. This approach considers only two different types of eNB and it also fails to consider the spatial distribution of UEs. Lung and Zhou proposed an agglomerative hierarchical clustering (AHC) two-type cell deployment (ATD) based on modified AHC (MAHC) to form macro and micro eNB clusters [19]. This approach utilizes micro eNBs to support the uncovered UEs. This approach fails to consider the uneven traffic demand from the hotspots. Han et al. proposed a traditional $K$-means HetNet deployment (TKD) approach by considering the uneven traffic demand from the hotspots [20]. This approach uses MAHC and $K$-means algorithms to form macro and micro eNB clusters. This approach additionally places pico eNBs to support the UEs in the dead areas and hotspots. All the above approaches failed to consider the limitations in terms of the radio resources.

Wang and Chuang proposed a four-stage eNB deployment with the minimum cost (EDMC) HetNet placement approach for LTE-A by considering the bandwidth and power constraints [4]. This approach effectively places and utilizes macro, micro, and pico eNBs to satisfy the coverage and traffic demands. In the first stage the required number of macro and micro eNBs is decided based on MAHC and weighted $K$-means algorithms to meet the coverage demand. During the second stage, macro eNBs are placed in the identified candidate sites. For every placed eNB, radio resource availability check is carried out in the third stage. Based on the results, some of the macro eNBs are made to micro eNBs by adjusting the transmitted power levels. To support the uncovered UEs pico eNBs are deployed in the fourth stage based on modified geometric disk cover (MGDC) algorithm. This approach effectively uses HetNet by properly distributing the traffic load among different eNBs. This approach also utilizes the path loss and shadowing models proposed in LTE Rel-8. From simulations, it is also clear that the approach proposed in [4] outperforms MCD, ATD [19], and TKD [20] approaches. There are few drawbacks for the aforementioned approach. The MAHC algorithm used in the first stage is computationally complex and iterative. The weighted $K$-means approach used to decide the required number of macro and micro eNBs needs $K$ values in advance. The MGDC algorithm used in the fourth stage for pico eNB placement can accommodate only two uncovered UEs [21].
This requires more number of pico eNBs. The capacity of pico eNBs is not fully exploited by the MGDC algorithm. This approach is also developed only for the coverage constraint.

In [5], a four-stage HetNet placement approach for $4 \mathrm{G}$ and beyond networks is proposed, which addressed some of the issues mentioned in [4]. This work utilizes fuzzy logic for macro, micro, and RS placement. The approach is proposed for coverage maximization. This approach also considers realistic path loss models proposed in 3GPP LTE-A. In [5], it is also proved that the computational complexity of fuzzy logic based approach is much lower than the other conventional approaches. But the placement cost minimization and pico eNB placement are not considered. The effect of interference on the performance of the proposed approach is also not investigated. The numerical results prove that the approach proposed in [5] offers improved system cost and power proportion performances over the conventional HetNet placement approaches.

In our proposed four-stage placement approaches, we have used fuzzy logic for the placement of macro, micro, pico, and RS nodes, while considering both the coverage and budget constraints together. Fuzzy logic based macro eNB selection and placement is carried out in the first stage. The power resource availability check and cell range adjustments are carried out in the second stage. To support the uncovered UEs, RSs or pico eNBs are placed in the third stage. In the fourth stage, power resource availability check is carried out for the placed RSs or pico eNBs. Under link overloading conditions, the services of few UEs are handed over to the neighbouring RSs or pico eNBs based on the availability of the radio resources. To minimize the inter-eNB and inter-RS interferences, this approach also uses $\mathrm{OI}$ as one of the inputs for FIE along with the service coverage index (SCI) and data traffic index (DTI).

The rest of the manuscript is organized in the following way: Section 2 presents the proposed system model and the fuzzy logic for eNB and RS placement. Section 3 presents the proposed three-input, fuzzy logic based four-stage HetNet placement approach for coverage and budget constraints. Section 4 validates the proposed approaches with the simulation results and Section 5 concludes the work by highlighting the future scopes.

\section{System Model}

The conventional $2 \mathrm{G}$ and $3 \mathrm{G}$ standards utilize eNBs with similar coverage [4]. LTE-A introduces the concept of HetNet, which supports eNBs with different coverage. LTE-A defines four different types of eNB, namely, macro, micro, pico, and femto, which all can coexist together in the same geographic region as shown in Figure 1. Macro, micro, and pico eNBs are operator deployed and needs a wired backhaul connection to the core network. Femto eNBs are deployed by the customer and it needs digital subscriber line (DSL) modem and internet connection for backhaul. The mobile switching center (MSC) in core network acts as a gateway between wired and wireless networks. All these eNBs share the same spectrum. The placement of more number of small 


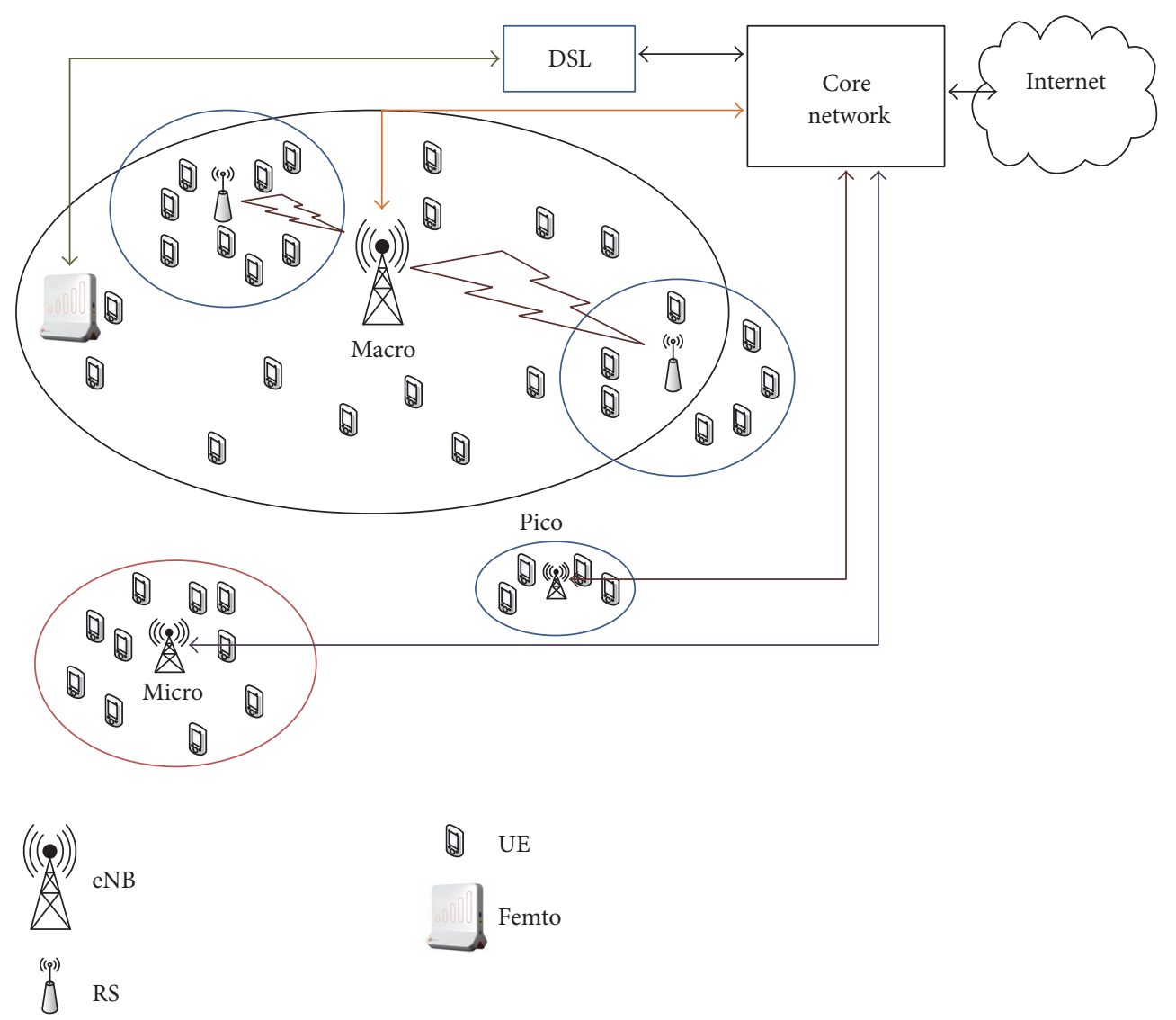

Figure 1: LTE-A HetNet and MHR architecture.

cells brings UEs closer to the serving eNBs. This minimizes the path loss and attenuation and increases the system capacity per unit area. To reduce the power consumption, the macro eNBs with less number of clients are made to microeNB by dynamically adjusting the transmitted power level. Pico eNBs are usually placed in the hotspots [22]. When there is a huge traffic demand from a particular area, the macro eNB offloads portion of the traffic demand to pico eNBs. Pico eNBs are more suited for outdoor applications. Femto eNBs are more suited for high capacity indoor applications. Since this work focuses on the eNB placement by the network operators, the placement of femto eNB is not considered here.

The UEs located near the cell boundary experience poor signal quality and data rate. IEEE $802.16 \mathrm{j}$ and LTE-A specifies relay technology for service coverage extension and capacity maximization $[23,24]$. RS is the most suited small cell solution for the coverage and capacity maximization problems, where the wired backhaul link is costly and unavailable. But it requires a backhaul link to its donor eNB. The spectrum used to access the UEs is also used by the RSs for backhaul connection. RSs utilize portion of the radio resources from its donar eNB. The coverage range, cost, and capacity of RS are larger than pico eNB. Hence, in this work, we utilize RS for service coverage maximization and pico eNB for placement cost minimization. The different types of eNB and RS defined in LTE-A are compared in Table 1.
2.1. Fuzzy Logic for HetNet and RS Placement. In this proposed approach, we have used the fuzzy logic to choose the placement sites of macro, micro, pico eNBs and RS from the available candidate sites. For the proposed Mamdani based fuzzy system, the input parameters are supplied as the crisp set. The crisp relation can be represented as [25]

$$
\psi_{A, B, C}(a, b, c)= \begin{cases}1, & (a, b, c) \in A, B, C, \\ 0, & (a, b, c) \notin A, B, C,\end{cases}
$$

where $\psi_{A, B, C}(a, b, c)$ is the crisp set $A$ with object $a$, set $B$ with object $b$, and set $C$ with object $c$ respectively. In crisp logic, if the object is available in the set, it is denoted as 1; otherwise it is 0 . The partial membership grading is not possible with crisp logic. Hence, in the fuzzy logic, for every value of sets $A$ and $B$, a fuzzy index is available within the interval $[0,1]$ which is denoted as $\psi_{A}(a): A \rightarrow[0,1], \psi_{B}(b): B \rightarrow[0,1]$. Similarly, for every value of the set $C$, a fuzzy index is available within the interval $[-1,1]$, which is denoted as $\psi_{C}(c): C \rightarrow[-1,1]$.

The SCI, OI, and DTI are the three input parameters to the FIE. Site selection index (SSI) is the output from the FIE. We have developed three trapezoidal Mamdani membership functions for SCI, OI, DTI, and SSI. Based on this, 27 rules are formulated. The defuzzifier is based on the centroid method [25]. The concept of SSI calculation from the FIE is illustrated in Figure 2. 
TABLE 1: Comparison of LTE-A eNBs and RS.

\begin{tabular}{|c|c|c|c|c|c|c|}
\hline Cell type & Location & Installation & Backhaul & Ideal cell radius & Number of users & PC \\
\hline Macro & Outdoor & Operator & Operator & $>1 \mathrm{~km}$ & $>256$ & $\sim \$ 444800$ \\
\hline Micro & Outdoor & Operator & Operator & $250 \mathrm{~m}-1 \mathrm{~km}$ & $32-200$ & $\sim \$ 47185$ \\
\hline Pico & Indoor/outdoor & Operator & Operator & $100 \mathrm{~m}-300 \mathrm{~m}$ & $32-64$ & $\sim \$ 13865$ \\
\hline Femto & Indoor & Customer & Customer & $10 \mathrm{~m}-50 \mathrm{~m}$ & $8-10$ & $\sim \$ 100$ \\
\hline $\mathrm{RS}$ & Indoor/outdoor & Operator & Operator & $250 \mathrm{~m}-1.125 \mathrm{~km}$ [9] & $32-120$ & $\sim \$ 62272$ \\
\hline
\end{tabular}

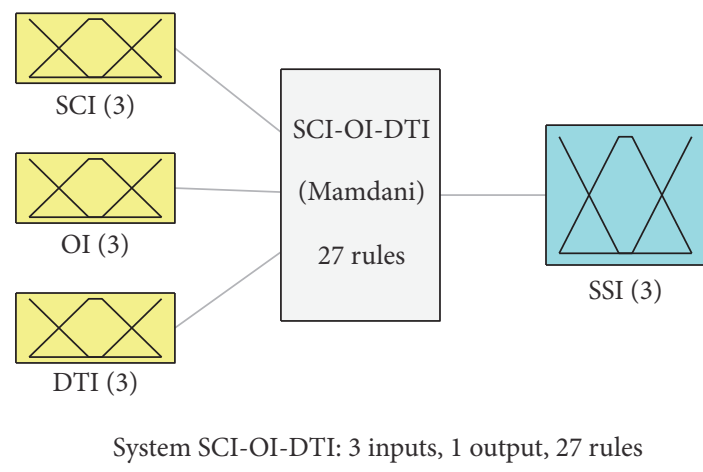

FIGURE 2: Block diagram of the proposed FIE.

The SCI of $k$ th candidate site is the ratio between the number of UEs covered by the eNB (macro, micro, and pico) placed in $k$ th site and the total number of UEs in the geographic region, which is given by [17]

$$
\mathrm{SCI}_{k}=\frac{S_{k}}{T},
$$

where $S_{k}$ is the number of UEs covered by the eNB placed in $k$ th candidate site and $T$ is the total number of UEs available in the geographic region.

The usage of single serving node requires huge radio resources to offer full network coverage over a given geographic area. It requires large transmission power to reach all potential users in that area. The limited radio resources available restrict the total number of users supported. This makes a single serving node supporting vast number of mobile users impractical. In order to increase the coverage and capacity and to use radio resources efficiently, the geographic area is divided into multiple cells. Each cell is supported by one serving node. Since the radio resources are reused at each cell, the total number of users supported is ideally scaled with the total number of deployed cells. The reduced distance between the serving node and UE increases the link quality yielding high signal to noise ratio (SNR). In homogeneous cell structure, the design specifications like cell size and transmit power budget allocated are common for all cells. This is not efficient especially in very dense, urban, and indoor environments in offering high throughput. In heterogeneous cell structure, small cells are overlapped with the conventional macrocell in both frequency and space.

When several serving nodes transmit their signals on the same frequency in the same geographic area, the UEs may not be able to distinguish to which serving node they are listening. The imperfect cell structures lead to severe inter cell interference at the cell edges. The interference reduces the link quality of cell edge UEs. This degrades the user experiencing data rate and increases the outages. The interference situation is unpredictable and more severe in HetNets. There exist two possible types of interference in small cells. The cotier interference occurs between the same cell types and the cross-tier interference occurs between different cell types. The cross-tier interference is difficult to control.

Sometimes small cells are expected to serve users even at low signal to interference plus noise ratio (SINR), which requires a strong mechanism to minimize or cope with interference. Fractional frequency reuse (FFR) can be used to minimize the interference by allocating different frequency bands for the closely located cells. But, the frequency and time domain resource allocation makes the FFR complex. The inter cell interference control (ICIC) feature is proposed in LTE Rel-10 with the specifications of high interference indication (HII), overload indication, and relative narrowband transmit power indicator (RNTP) signaling between eNBs. RNTP signaling coordinates the downlink transmitted power between the cells to simplify FFR for data channels. But this feature cannot deal with the interference to/from reference signals and control channels. In contrast, serving nodes that are adequately far from each other may reuse the same frequency bands with less interference. Hence in this work, to minimize the cotier and cross-tier interferences, the serving nodes are placed adequately far from each other. This minimizes the OI between the cells and maintains the link quality even at the cell edges. Many of the conventional HetNet placement approaches have not considered the interference minimization problem. In this work, we are addressing the interference problem in the serving node placement stage itself. The OI of eNB available in the $k$ th candidate site is given by [26]

$$
=\frac{y^{2} \cos ^{-1}\left(\left(\delta^{2}+y^{2}-x^{2}\right) / 2 \delta y\right)+x^{2} \cos ^{-1}\left(\left(\delta^{2}+x^{2}-y^{2}\right) / 2 \delta x\right)-(1 / 2) \sqrt{(-\delta+y+x)(\delta+y-x)(\delta-y+x)(\delta+y+x)}}{\pi y^{2}}
$$


where the numerator is the overlapping area, $x$ and $y$ are the radius of two overlapping cells, and $\delta$ is the distance between the center points of the overlapped cells.

The average information rate of the UEs covered by the eNB placed in $k$ th candidate site is given by

$$
\beta_{d, k}=\frac{1}{S_{k}} \sum_{l=1}^{S_{k}} T_{l},
$$

where $T_{l}$ is the data rate of $l$ th UE. The DTI of $k$ th candidate site is given by [17]

$$
\mathrm{DTI}_{k}=\frac{\beta_{d, k}-\beta_{t, k}}{\max \left\{\beta_{d, k}, \beta_{t, k}\right\}},
$$

where $\beta_{t, k}$ is the average traffic demands of the UEs covered by the eNB placed in $k$ th candidate site.

The fuzzy sets for SCI of $k$ th candidate site take the linguistic variables like Low, Medium, and High. These are presented below by the membership functions $\psi_{A_{1}}^{k}(a)$, $\psi_{A_{2}}^{k}(a)$, and $\psi_{A_{3}}^{k}(a)$, respectively, over the interval $[0,1]$ :

$$
\begin{aligned}
& \psi_{A_{1}}^{k}(a)= \begin{cases}1, & a \leq 0, \\
\frac{(0.45-a)}{0.45}, & 0<a<0.45, \\
0, & a \geq 0.45,\end{cases} \\
& \psi_{A_{2}}^{k}(a)= \begin{cases}0, & a \leq 0, \\
\frac{a}{0.45}, & 0<a<0.45, \\
1, & 0.45<a<0.55, \\
\frac{(1-a)}{0.45}, & 0.55<a<1, \\
0, & a \geq 1,\end{cases} \\
& \psi_{A_{3}}^{k}(a)= \begin{cases}0, & a \leq 0.55, \\
\frac{(a-0.55)}{0.45}, & 0.55<a<1, \\
1, & a \geq 1,\end{cases}
\end{aligned}
$$

where $a \in[0,1]$. The membership function diagram of SCI is shown in Figure 3.

The fuzzy sets for OI of $k$ th candidate site take the linguistic variables like Less, Moderate, and More. These are presented below by the membership functions $\psi_{B_{1}}^{k}(b), \psi_{B_{2}}^{k}(b)$, and $\psi_{B_{3}}^{k}(b)$, respectively, over the interval $[0,1]$ :

$$
\psi_{B_{1}}^{k}(b)= \begin{cases}1, & b \leq 0, \\ \frac{(0.45-b)}{0.45}, & 0<b<0.45, \\ 0, & b \geq 0.45,\end{cases}
$$

$$
\begin{gathered}
\psi_{B_{2}}^{k}(b)= \begin{cases}0, & b \leq 0, \\
\frac{b}{0.45}, & 0<b<0.45, \\
1, & 0.45<b<0.55, \\
\frac{(1-b)}{0.45}, & 0.55<b<1, \\
0, & b \geq 1,\end{cases} \\
\psi_{B_{3}}^{k}(b)= \begin{cases}0, & b \leq 0.55, \\
\frac{(b-0.55)}{0.45}, & 0.55<b<1, \\
1, & b \geq 1,\end{cases}
\end{gathered}
$$

where $b \in[0,1]$. The membership function diagram of $\mathrm{OI}$ is shown in Figure 4.

The fuzzy sets for DTI of $k$ th candidate site take the linguistic variables like Negative, Center, and Positive. These are presented below by the membership functions $\psi_{\mathrm{C}_{1}}^{k}(c)$, $\psi_{\mathrm{C}_{2}}^{k}(c)$, and $\psi_{\mathrm{C}_{3}}^{k}(c)$, respectively, over the interval $[-1,1]$ :

$$
\begin{aligned}
& \psi_{C_{1}}^{k}(c)= \begin{cases}1, & c \leq-1, \\
\frac{(-0.1-c)}{0.9}, & -1<c<-0.1, \\
0, & c \geq-0.1,\end{cases} \\
& \psi_{C_{2}}^{k}(c)= \begin{cases}0, & c \leq-1, \\
\frac{(c+1)}{0.9}, & -1<c<-0.1, \\
1, & -0.1<c<0.1, \\
\frac{(1-c)}{0.9}, & 0.1<c<1, \\
0, & c \geq 1,\end{cases} \\
& \psi_{C_{3}}^{k}(c)= \begin{cases}0, & c \leq 0.1, \\
\frac{(c-0.1)}{0.9}, & 0.1<c<1, \\
1, & c \geq 1,\end{cases}
\end{aligned}
$$

where $c \in[-1,1]$. The membership function diagram of DTI is shown in Figure 5.

The SSI of each candidate site is taken as the output from the FIE. The linguistic variables for the SSI of $k$ th candidate site are Poor, Average, and Good. These are represented below by the membership functions $\psi_{D_{1}}^{k}(d), \psi_{D_{2}}^{k}(d)$, and $\psi_{D_{3}}^{k}(d)$, respectively, over the interval $[-1,1]$ :

$$
\psi_{D_{1}}^{k}(d)= \begin{cases}1, & d \leq-1 \\ \frac{(-0.1-d)}{0.9}, & -1<d<-0.1 \\ 0, & d \geq-0.1\end{cases}
$$




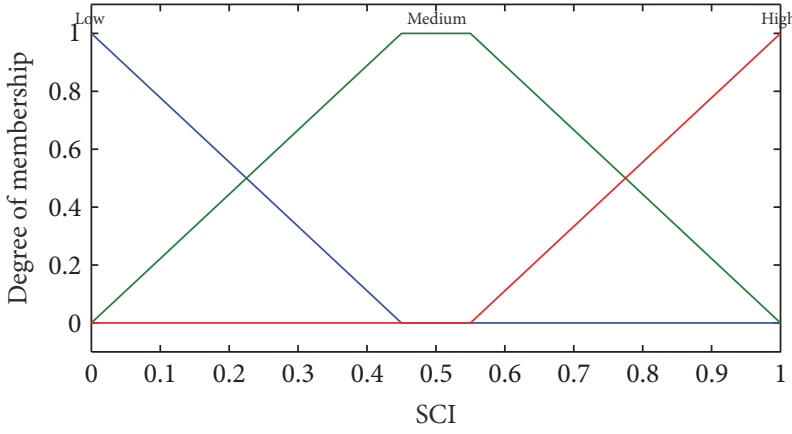

FIGURE 3: The membership function diagram of SCI.

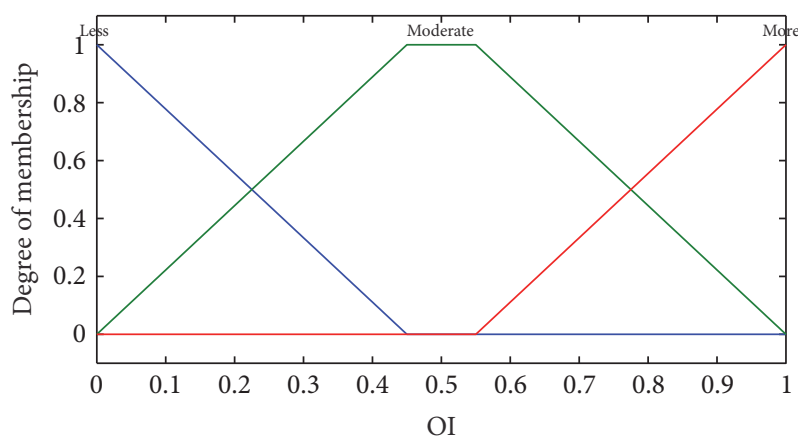

FIGURE 4: The membership function diagram of OI.

$$
\begin{aligned}
& \psi_{D_{2}}^{k}(d)= \begin{cases}0, & d \leq-1, \\
\frac{(d+1)}{0.9}, & -1<d<-0.1 \\
1, & -0.1<d<0.1, \\
\frac{(1-d)}{0.9}, & 0.1<d<1, \\
0, & d \geq 1,\end{cases} \\
& \psi_{D_{3}}^{k}(d)= \begin{cases}0, & d \leq 0.1, \\
\frac{(d-0.1)}{0.9}, & 0.1<d<1, \\
1, & d \geq 1,\end{cases}
\end{aligned}
$$

where $d \in[-1,1]$. The membership function diagram of SSI is shown in Figure 6.

The input and output fuzzy sets described above corresponded to delivering the rules for the FIE. The fuzzy rules framed for the FIE are described as follows.

Rule 1. If $\mathrm{SCI}_{k}$ is Low and $\mathrm{OI}_{k}$ is Less and $\mathrm{DTI}_{k}$ is Negative then $\mathrm{SSI}_{k}$ is Poor.

Rule 2. If $\mathrm{SCI}_{k}$ is Low and $\mathrm{OI}_{k}$ is Less and $\mathrm{DTI}_{k}$ is Center then $\mathrm{SSI}_{k}$ is Average.

Rule 3. If $\mathrm{SCI}_{k}$ is Low and $\mathrm{OI}_{k}$ is Less and $\mathrm{DTI}_{k}$ is Positive then $\mathrm{SSI}_{k}$ is Good.

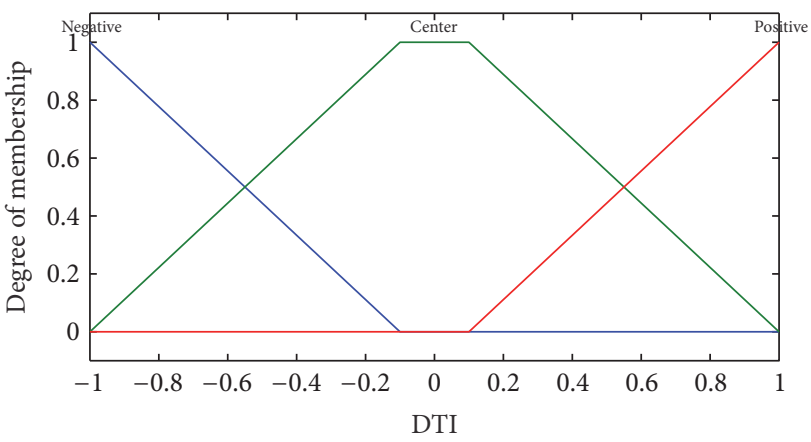

FIGURE 5: The membership function diagram of DTI.

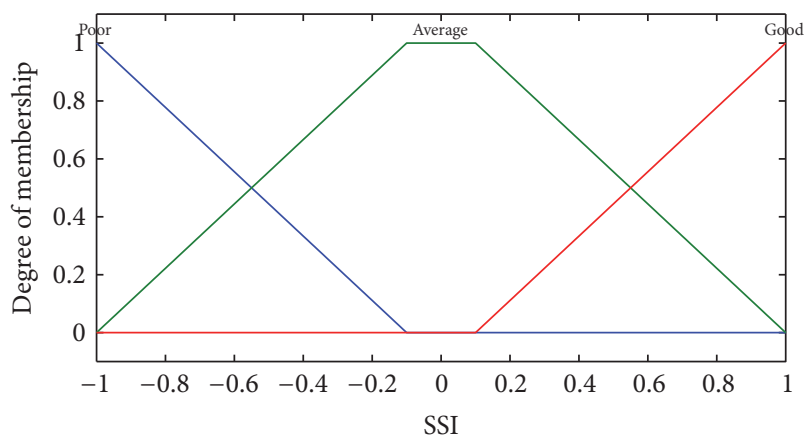

FIGURE 6: The membership function diagram of SSI.

Rule 4. If $\mathrm{SCI}_{k}$ is Low and $\mathrm{OI}_{k}$ is Moderate and $\mathrm{DTI}_{k}$ is Negative then $\mathrm{SSI}_{k}$ is Poor.

Rule 5. If $\mathrm{SCI}_{k}$ is Low and $\mathrm{OI}_{k}$ is Moderate and $\mathrm{DTI}_{k}$ is Center then $\mathrm{SSI}_{k}$ is Average.

Rule 6. If $\mathrm{SCI}_{k}$ is Low and $\mathrm{OI}_{k}$ is Moderate and $\mathrm{DTI}_{k}$ is Positive then $\mathrm{SSI}_{k}$ is Average.

Rule 7. If $\mathrm{SCI}_{k}$ is Low and $\mathrm{OI}_{k}$ is More and $\mathrm{DTI}_{k}$ is Negative then $\mathrm{SSI}_{k}$ is Poor.

Rule 8. If $\mathrm{SCI}_{k}$ is Low and $\mathrm{OI}_{k}$ is More and $\mathrm{DTI}_{k}$ is Center then $\mathrm{SSI}_{k}$ is Poor.

Rule 9. If $\mathrm{SCI}_{k}$ is Low and $\mathrm{OI}_{k}$ is More and $\mathrm{DTI}_{k}$ is Positive then $\mathrm{SSI}_{k}$ is Poor.

Rule 10. If $\mathrm{SCI}_{k}$ is Medium and $\mathrm{OI}_{k}$ is Less and $\mathrm{DTI}_{k}$ is Negative then $\mathrm{SSI}_{k}$ is Poor.

Rule 11. If $\mathrm{SCI}_{k}$ is Medium and $\mathrm{OI}_{k}$ is Less and $\mathrm{DTI}_{k}$ is Center then $\mathrm{SSI}_{k}$ is Average.

Rule 12. If $\mathrm{SCI}_{k}$ is Medium and $\mathrm{OI}_{k}$ is Less and $\mathrm{DTI}_{k}$ is Positive then $\mathrm{SSI}_{k}$ is Good.

Rule 13. If $\mathrm{SCI}_{k}$ is Medium and $\mathrm{OI}_{k}$ is Moderate and $\mathrm{DTI}_{k}$ is Negative then $\mathrm{SSI}_{k}$ is Poor. 
Rule 14. If $\mathrm{SCI}_{k}$ is Medium and $\mathrm{OI}_{k}$ is Moderate and $\mathrm{DTI}_{k}$ is Center then $\mathrm{SSI}_{k}$ is Average.

Rule 15. If $\mathrm{SCI}_{k}$ is Medium and $\mathrm{OI}_{k}$ is Moderate and $\mathrm{DTI}_{k}$ is Positive then $\mathrm{SSI}_{k}$ is Good.

Rule 16. If $\mathrm{SCI}_{k}$ is Medium and $\mathrm{OI}_{k}$ is More and $\mathrm{DTI}_{k}$ is Negative then $\mathrm{SSI}_{k}$ is Poor.

Rule 17. If $\mathrm{SCI}_{k}$ is Medium and $\mathrm{OI}_{k}$ is More and $\mathrm{DTI}_{k}$ is Center then $\mathrm{SSI}_{k}$ is Average.

Rule 18. If $\mathrm{SCI}_{k}$ is Medium and $\mathrm{OI}_{k}$ is More and $\mathrm{DTI}_{k}$ is Positive then $\mathrm{SSI}_{k}$ is Good.

Rule 19. If $\mathrm{SCI}_{k}$ is High and $\mathrm{OI}_{k}$ is Less and $\mathrm{DTI}_{k}$ is Negative then $\mathrm{SSI}_{k}$ is Good.

Rule 20. If $\mathrm{SCI}_{k}$ is High and $\mathrm{OI}_{k}$ is Less and $\mathrm{DTI}_{k}$ is Center then $\mathrm{SSI}_{k}$ is Good.

Rule 21. If $\mathrm{SCI}_{k}$ is High and $\mathrm{OI}_{k}$ is Less and $\mathrm{DTI}_{k}$ is Positive then $\operatorname{SSI}_{k}$ is Good.

Rule 22. If $\mathrm{SCI}_{k}$ is High and $\mathrm{OI}_{k}$ is Moderate and $\mathrm{DTI}_{k}$ is Negative then $\mathrm{SSI}_{k}$ is Average.

Rule 23. If $\mathrm{SCI}_{k}$ is High and $\mathrm{OI}_{k}$ is Moderate and $\mathrm{DTI}_{k}$ is Center then $\mathrm{SSI}_{k}$ is Average.

Rule 24. If $\mathrm{SCI}_{k}$ is High and $\mathrm{OI}_{k}$ is Moderate and $\mathrm{DTI}_{k}$ is Positive then $\mathrm{SSI}_{k}$ is Good.

Rule 25. If $\mathrm{SCI}_{k}$ is High and $\mathrm{OI}_{k}$ is More and $\mathrm{DTI}_{k}$ is Negative then $\mathrm{SSI}_{k}$ is Poor.

Rule 26. If $\mathrm{SCI}_{k}$ is High and $\mathrm{OI}_{k}$ is More and $\mathrm{DTI}_{k}$ is Center then $\mathrm{SSI}_{k}$ is Average.

Rule 27. If $\mathrm{SCI}_{k}$ is High and $\mathrm{OI}_{k}$ is More and $\mathrm{DTI}_{k}$ is Positive then $\mathrm{SSI}_{k}$ is Good.

In Figure 7, the calculation of SSI from SCI, OI, and DTI is illustrated with the help of rule viewer diagram. For example, consider $\mathrm{SCI}_{k}$ of $0.7, \mathrm{OI}_{k}$ of 0.5 , and $\mathrm{DTI}_{k}$ of 0.5 . The membership functions of $\mathrm{SCI}_{k}$ for Low, Medium, and $H i g h$ values are $0,0.75$, and 0.4 , respectively. The membership functions of $\mathrm{OI}_{k}$ for Less, Moderate, and More values are 0 , 0.9 , and 0 , respectively. Similarly, the membership functions of $\mathrm{DTI}_{k}$ for Negative, Center, and Positive values are 0, 0.7, and 0.5 , respectively. These conditions fall under Rules 14 , 15,23 , and 24 , respectively. The defuzzified output parameter value for $\mathrm{SSI}_{k}$ is identified as 0.088 . The variations of the crisp values connected with the SSI with respect to any two of the three fuzzy inputs are shown in Figures 8-10 using surface plots. It is clear that the inputs and output in the surface plots obey the developed rules. In all the input output fuzzy sets developed above, their membership functions and the developed rules are applicable for RSs and pico eNBs.

\section{The Proposed HetNet and MHR Placement Approaches}

The proposed approaches should choose the diverse eNB and RS placement sites based on the coverage and budget constraints to meet the following objectives: Each UE in the given geographic area should have a continuous high quality connection with an eNB or a RS. The traffic demands of UEs should be satisfied by properly allocating the radio resources. The proposed approach should also balance the traffic load between different eNBs and RSs so that the total power consumption and network traffic overloading get minimized. The network PC and the interference between the adjacent placed cells should be minimized. In order to meet the coverage and cost requirements, we have proposed two different approaches, which are explained below in detail.

3.1. SSMSC Approach. This approach should identify the required number of serving nodes, their types, and the placement locations to achieve the SCI higher than the target SCI. As mentioned in Table 1, the coverage range of RS is larger than pico eNB. Hence, in SSMSC approach, the placement of RSs is entertained to maximize the coverage.

This approach is executed in four stages to satisfy the following objectives:

$$
\frac{1}{T}\left[\sum_{k=1}^{E} \alpha_{k} S_{k}+\sum_{n=1}^{R} \beta_{n} S_{n}\right] \geq T_{\mathrm{SCI}},
$$

with the binary valued functions

$$
\begin{aligned}
& \alpha_{k}= \begin{cases}1, & \text { if macro or micro } \mathrm{eNB}_{k} \text { is placed, } \\
0, & \text { else, }\end{cases} \\
& \beta_{n}= \begin{cases}1, & \text { if } \mathrm{RS}_{n} \text { is placed, } \\
0, & \text { else, }\end{cases}
\end{aligned}
$$

where $E$ and $R$ are the number of potential candidate sites for eNB and RS placement, respectively. $T_{\mathrm{SCI}}$ and $S_{n}$ are the target SCI and number of UEs covered by $n$th RS, respectively.

The abovementioned approach is executed under the following constraints:

(1) Power constraint is

$$
\begin{array}{r}
\sum_{s_{l}^{k} \in T} p_{k, l}+\sum_{n=1}^{N^{k}} p_{k, n} \beta_{k, n} \leq P_{k} \alpha_{k}, \\
\sum_{s_{r}^{n} \in T} p_{n, r}^{k} \leq P_{n} \beta_{n}
\end{array}
$$

$$
\forall k \in \mathbf{E}, n \in \mathbf{R},(l, r) \in \mathbf{T} \text {, }
$$

where $P_{k}$ and $P_{n}$ are the aggregate power allocated to $k$ th $\mathrm{eNB}$ and $n$th RS, respectively, $s_{l}^{k}$ is the $l$ th UE covered by $k$ th eNB, and $p_{k, l}$ and $p_{k, n}$ are the transmitted power allocated to $l$ th UE and $n$th RS by 

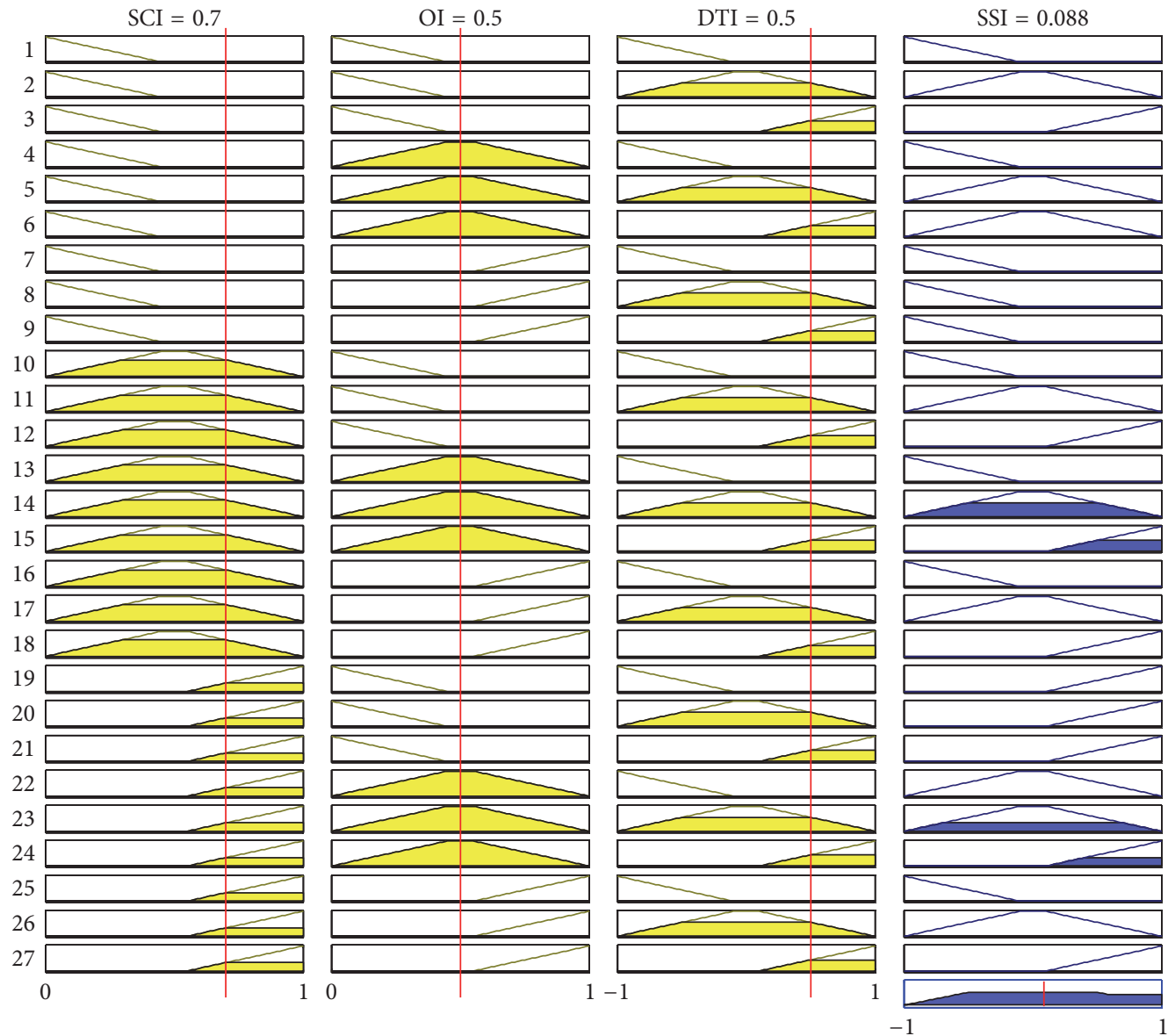

FIgURE 7: Rule viewer diagram illustrating the SSI calculation.

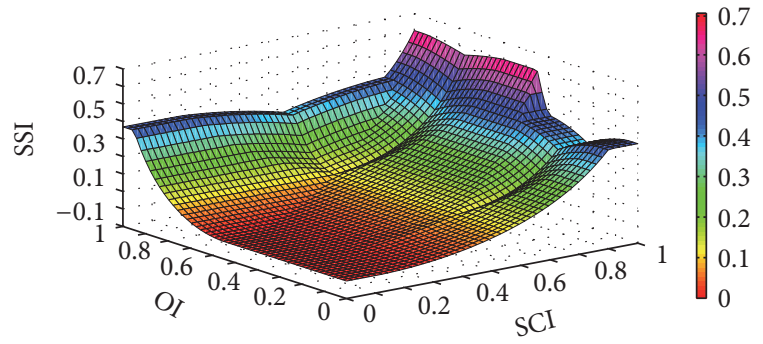

FIGURE 8: Surface plot of SSI versus SCI-OI combinations.

$k$ th eNB, respectively. $N^{k}$ is the selected candidate sites of RS within the range of $k$ th eNB, $s_{r}^{n}$ is the $r$ th UE covered by $n$th RS, and $p_{n, r}^{k}$ is the transmitted power allocated to $r$ th UE by $n$th RS in $k$ th eNB. E and $\mathbf{R}$ are the set of candidate sites for macro eNB and RS, respectively. $\mathbf{T}$ is the coordinates of UEs in the given geographic area.

(2)

$$
p_{k, l}, p_{k, n}, p_{n, r}^{k} \geq 0
$$

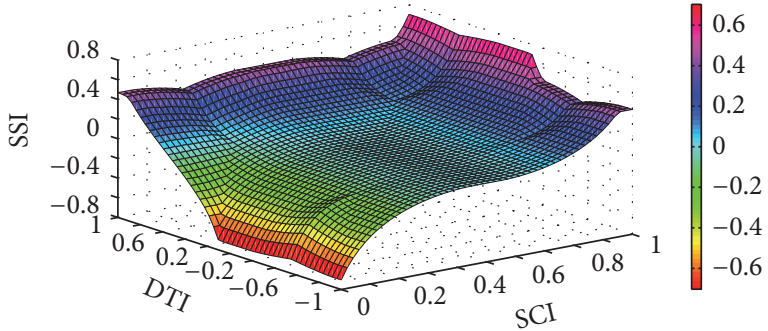

FIgURE 9: Surface plot of SSI versus SCI-DTI combinations.

(3) The OI for the eNB and RS should be less than the OI threshold $(I)$ :

$$
\mathrm{OI}^{\mathrm{eNB}}, \mathrm{OI}^{\mathrm{RS}}<I .
$$

To meet the expected service coverage under the above constraints, the proposed approach is executed in four stages.

3.1.1. Stage 1: Candidate Site Selection and Placement of Macro eNBs. The procedure for SSMSC based candidate site selection and placement of macro eNBs is shown in Algorithm 1. The set of potential candidate sites for macro eNB, set of UEs covered by $k$ th macro eNB, and target SCI are given as the 


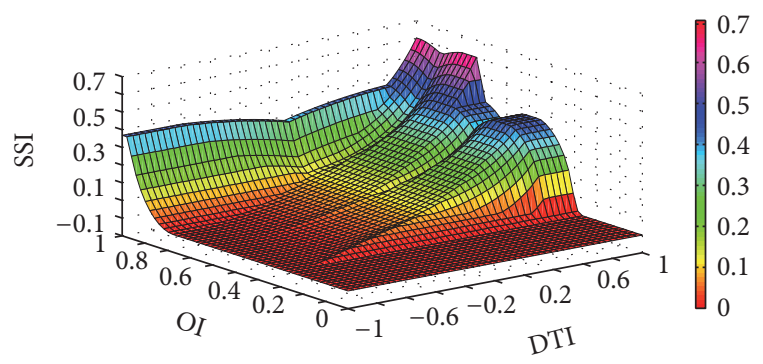

FIgURE 10: Surface plot of SSI versus DTI-OI combinations.

input to this stage. The identified candidate sites of macro eNB are the output from this stage. The SCI is initialized to zero. SCI, OI, and DTI of each eNB candidate site are given as the input parameters for FIE and the corresponding output parameter SSI is obtained in steps (2) and (3), respectively. The candidate site with maximum SSI is identified in step (6). Based on this, SCI is upgraded in step (7). If the target SCI is not achieved, the coordinates of identified candidate site with maximum SSI are stored in Sel_eNB. The corresponding SSI is made to zero in step (13), so that, in step (6), the next highest SSI candidate site will be chosen for the next iteration. This procedure is rehashed until the condition in step (8) gets fulfilled. All the recognized macro eNB candidate sites with highest SSI are stored in Sel_eNB.

3.1.2. Stage 2: Power Resource Availability Check and Micro eNB Placement. The target downlink information transmission rate from $k$ th eNB to $l$ th UE is given by [4]

$$
d_{k, l}^{\mathrm{DL}}=B_{k, l}^{\mathrm{DL}} \log _{2}\left(1+\frac{p_{k, l}^{r}\left|h_{k, l}\right|^{2}}{B_{k, l}^{\mathrm{DL}} \gamma N_{0}}\right),
$$

where $\left|h_{k, l}\right|^{2}$ is the multipath fading channel gain, $N_{0}$ is the noise power, $\gamma$ is the SNR gap, and $B_{k, l}^{\mathrm{DL}}$ is the bandwidth allocated to $l$ th UE by $k$ th eNB. To obtain the target downlink rate, the minimum required received signal power is given by

$$
p_{k, l}^{r}=\left(2^{d_{k, l}^{\mathrm{DL}} / B_{k, l}^{\mathrm{DL}}}-1\right)\left(\frac{B_{k, l}^{\mathrm{DL}} \gamma N_{0}}{\left|h_{k, l}\right|^{2}}\right) .
$$

The required transmit power from $k$ th eNB to $l$ th UE to meet the target downlink rate is given by

$$
p_{k, l}(\mathrm{~dB})=p_{k, l}^{r}(\mathrm{~dB})+\mathrm{PL}(\mathrm{dB}), \quad \mathrm{PL}(\mathrm{dB}) \in\left\{\mathrm{PL}_{\text {macro eNB }}(\mathrm{dB}), \mathrm{PL}_{\text {micro eNB }}(\mathrm{dB}), \mathrm{PL}_{\text {pico eNB }}(\mathrm{dB}), \mathrm{PL}_{\mathrm{RS}}(\mathrm{dB})\right\}
$$

where $\mathrm{PL}_{\text {macro eNB }}(\mathrm{dB}), \mathrm{PL}_{\text {micro eNB }}(\mathrm{dB})$, and $\mathrm{PL}_{\text {pico eNB }}(\mathrm{dB})$ are the transmission path loss in $\mathrm{dB}$ for macro, micro, and pico eNBs, which are given by $[27,28]$

$$
\mathrm{PL}_{\text {macro eNB }}(\mathrm{dB})=128.1+37.6 \log _{10}(v) \text {, }
$$

where $v$ is the distance from eNB to UE or RS.

$$
\begin{aligned}
\mathrm{PL}_{\text {micro eNB }}(\mathrm{dB}) & =140.7+37.6 \log _{10}(v) \\
& =\mathrm{PL}_{\text {pico eNB }}(\mathrm{dB}) .
\end{aligned}
$$

The transmission path loss for RS in $\mathrm{dB}$ is given by [23]

$$
\mathrm{PL}_{\mathrm{RS}}(\mathrm{dB})=103.8+20.9 \log _{10}(v) .
$$

The idea of SSMSC based power resource availability check in macro eNB and micro eNB placement is illustrated in Algorithm 2. The selected candidate sites of macro eNB from the first stage, noise power, SNR gap, target downlink information transmission rate, bandwidth supported per UE, UEs supported by $k$ th macro eNB, and maximum RS capacity $(u)$ are the inputs to this stage. Identified macro and micro eNBs for placement and UEs that are not covered by any of the placed eNBs $(\widehat{S})$ are the outputs from this stage.

In step (2), the transmit power required to serve all UEs covered by $k$ th eNB $\left(p_{k}\right)$ is initialized to zero. In step (4), the distance between $k$ th eNB and $l$ th UE is calculated. Based on the identified distance, path loss and transmit power required to meet the target data rate are obtained using steps (5) and (6), respectively. This methodology is rehashed for all UEs covered under $k$ th $\mathrm{eNB}$. The aggregate power required to bolster all these UEs is calculated in step (7). If the aggregate power required by $k$ th eNB exceeds $P_{\text {macro eNB }}$, then the outermost UEs are identified and expelled from the present serving eNB one by one, until the power constraint in (12) gets fulfilled. This is done between steps (9) and (14). The expelled UEs are stored in $\widehat{S}$ and their related distances are made to zero. This procedure is rehashed for all recognized macro eNBs.

When all the recognized macro eNBs fulfill the power constraint, the idea of cell shrinking is carried out, which is outlined in Algorithm 3. In step (1), the aggregate power required to bolster all UEs under each macro eNB is compared with the $P_{\text {micro enB }}$. If it is true, the corresponding macrocell is shrunk to microcell by dynamically adjusting the power level. This is done in step (2). Due to cell shrinking, some of the UEs in the cell edges become uncovered. On the off chance that the condition given in step (1) is not met, then discard the service for some of the outermost UEs and revalidate the condition. Still, if the condition is not satisfied, do not go for cell shrinking. The procedure of expelling the outermost UEs ought to be done just for certain predefined number of UEs, which is considered in step (3).

3.1.3. Stage 3: Candidate Site Selection and Placement of RSs. The procedure for SSMSC based candidate site selection and placement of RSs is outlined in Algorithm 4. The SCI is upgraded after the completion of second stage and given as one of the inputs for stage 3. The set of candidate sites 


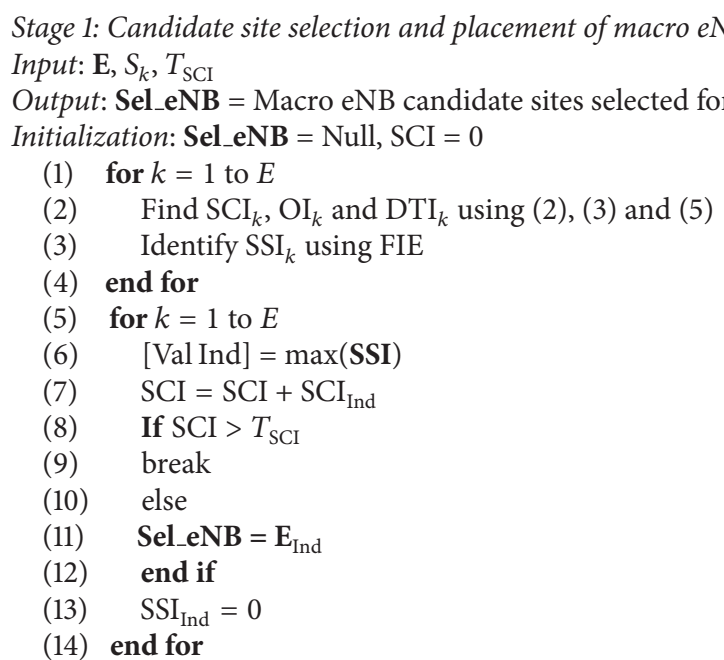

Algorithm 1: Candidate site selection and placement of macro eNBs for SSMSC approach.

Stage 2: Power resource availability check in macro eNB and micro eNB placement Input: Sel_eNB, $N_{0}, \gamma, d_{k, l}^{\mathrm{DL}}, B_{k, l}^{\mathrm{DL}}, S_{k}, u$

Output: Sites identified for macro and micro eNBs placement, $\widehat{S}$

(1) for $k=1$ to length(Sel_eNB)

(2) $\quad p_{k}=0$

(3) for $l=1$ to $S_{k}$

(4) $\quad v(l)=\mid \operatorname{Sel} \_$eNB $(k)-s_{l} \mid$

(5) Substitute $v(l)$ in (19) to obtain the PL

(6) Calculate $p_{k, l}$ using (18)

(7) $\quad p_{k}=p_{k}+p_{k, l}$

(8) end for

(9) If $p_{k}>P_{\text {macro eNB }}$

(10) $\quad$ [Val Ind $]=\max (\mathbf{v})$

(11) $\quad p_{k}=p_{k}-p_{\text {Ind }}$

(12) $\quad \widehat{S} \leftarrow s_{\text {Ind }}$

(13) $\quad \mathbf{v}($ Ind $)=0$

(14) end if

(15) Repeat steps (9) to (14) until (12) gets satisfied

(16) Cell shrinking $\left(\mathbf{p}_{\mathbf{k}}\right)$

(17) end for

Algorithm 2: Power resource availability check in macro eNB and micro eNB placement for SSMSC approach.

Cell shrinking $\left(\mathbf{p}_{\mathbf{k}}\right)$

(1) If $p_{k} \leq P_{\text {micro eNB }}$

(2) $\quad \mathbf{E}_{k} \rightarrow P_{\text {micro eNB }}$

(3) else if $\left(S_{k}-M_{k}\right) \leq u$ where $M_{k}$ is the number of served UEs by $k$ th micro eNB

(4) [Val Ind $]=\max (\mathbf{v})$

(5) $\quad p_{k}=p_{k}-p_{\text {Ind }}$

(6) $\widehat{S} \leftarrow s_{\text {Ind }}$

(7) $\quad \mathbf{v}($ Ind $)=0$

(8) end if

(9) Repeat step (4) to (7), until (12) gets satisfied

Algorithm 3: The idea of cell shrinking. 


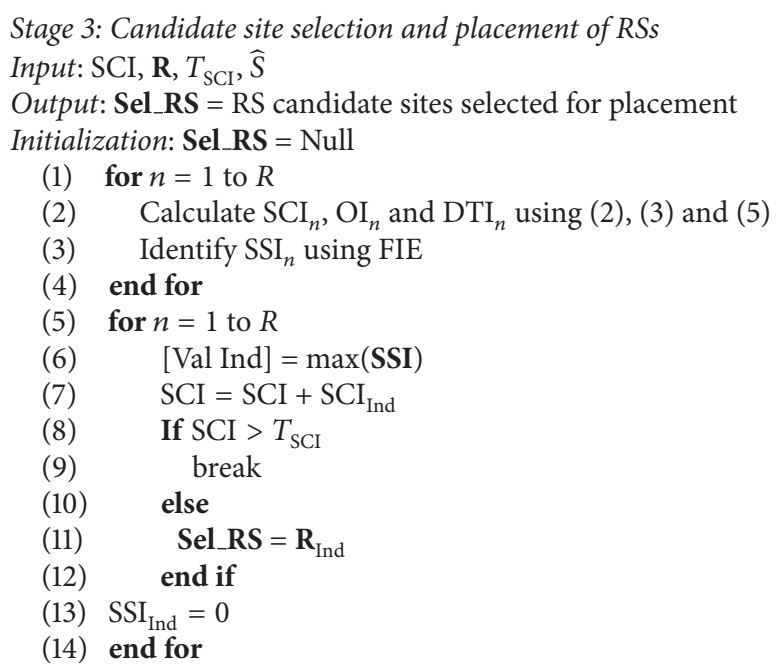

Algorithm 4: Candidate site selection and placement of RSs.

for RS placement, target SCI, and the set of UEs that are not covered by any of the placed eNBs are also given as the input to this stage. The recognized candidate sites of RS are the output from this stage. SCI, OI, and DTI of each RS candidate site are given as the input parameters for FIE and the corresponding output parameter SSI is obtained in steps (2) and (3), respectively. The candidate site with maximum SSI is recognized in step (6). Based on this, SCI is upgraded in step (7). If the target SCI is not achieved, the coordinates of identified candidate site with maximum SSI are stored in Sel_RS. In step (13), the corresponding SSI is made to zero, so that, in step (6), the next highest SSI candidate site will be chosen for the next iteration. This procedure is rehashed until the condition in step (8) gets fulfilled. All the recognized RS candidate sites with highest SSI are stored in Sel_RS.

3.1.4. Stage 4: Power Resource Availability Check for RSs and RS Selection. The idea of SSMSC based power resource availability check for the placed RSs and RS selection to satisfy UEs service demand is outlined in Algorithm 5. The selected candidate sites of RSs from the third stage, noise power, SNR gap, expected downlink information transmission rate $\left(d_{n, r}^{\mathrm{DL}}\right)$, bandwidth allocated per UE by $n$th $\mathrm{RS}\left(B_{n, r}^{\mathrm{DL}}\right)$, and UEs supported by $n$th RS $\left(S_{n}\right)$ are the inputs to this stage. The RSs selected to offer requested service demand are the outputs from this stage. In step (2), the transmit power required to serve all UEs covered by $n$th RS $\left(p_{n}\right)$ is initialized to zero. In step (4), the distance between $n$th RS and $r$ th UE is calculated. Based on the identified distance, path loss and transmit power required to meet the target data rate are obtained using steps (5) and (6), respectively. The transmit power required can be obtained by using equations similar to (16)-(18). This methodology is rehashed for all UEs covered under $n$th RS.
The aggregate power required to bolster all these UEs is calculated in step (7).

If the aggregate power required by $n$th eNB exceeds $P_{\mathrm{RS}}$, then the outermost UEs are identified and expelled from present serving RS one by one, until the power constraint in (13) gets fulfilled. This process is repeated for all selected RSs. When all the selected RSs satisfy the power constraint, the idea of RS selection is carried out by the donor macro eNB. The services of UEs, which are discarded by the current serving RS, are handed over to the neighboring RS based on the availability of the radio resources. This process minimizes the link traffic overloading.

3.2. SSMPC Approach. This approach should identify the required number of eNBs, their types, and the placement locations to maximize the service coverage and power proportion by maintaining the PC less than the total placement budget (TPB). As mentioned in Table 1, the PC of pico eNB is lesser than RS. Hence, in SSMPC approach, to bolster the traffic demand from hotspots and dead areas, the placement of pico eNBs is entertained.

The SSMPC problem is outlined as [9]

$$
\sum_{k=1}^{E} \alpha_{k} \cdot C_{k}+\sum_{m=1}^{B} \mu_{m} \cdot C_{\text {pico }} \leq \mathrm{TPB},
$$

with the binary variable

$$
\mu_{m}= \begin{cases}1, & \text { if pico } \mathrm{eNB}_{m} \text { is placed } \\ 0, & \text { else }\end{cases}
$$

where $B$ represents the total number of candidate sites for pico eNB and $C_{k}$ represents the PC (installation and 


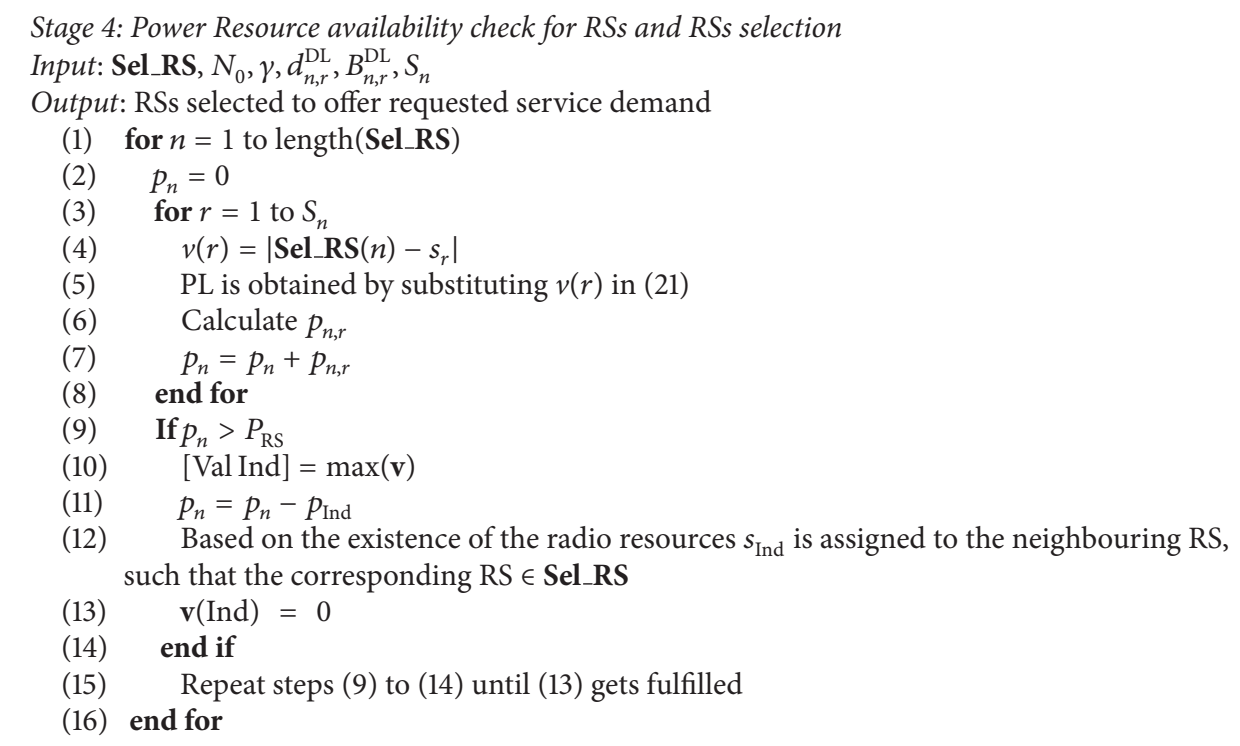

Algorithm 5: Power resource availability check for RSs and RSs selection.

maintenance) of macro and micro eNBs, respectively. $C_{\text {pico }}$ represents the PC of pico eNB.

$$
C_{k} \in\left\{C_{\text {macro eNB }}, C_{\text {micro eNB }}\right\} \text {, }
$$

where $C_{\text {macro eNB }}$ and $C_{\text {micro eNB }}$ denote the PC of macro eNB and micro eNB, respectively.

The abovementioned approach is executed under the following constraints:

(1) Power constraint is

$$
\begin{aligned}
& \sum_{s_{l} \in T} p_{k, l} \leq P_{k} \cdot \alpha_{k}, \\
& \sum_{s_{q} \in T} p_{m, q} \leq P_{m} \cdot \mu_{m}, \quad \forall k \in \mathbf{E}, m \in \mathbf{B},(l, q) \in \mathbf{T},
\end{aligned}
$$

where $P_{m}$ represents the total power allocated to $m$ th pico eNB, $s_{q}$ represents the $q$ th UE covered by $m$ th pico eNB, and $p_{m, q}$ is the transmit power allocated to $q$ th UE by $m$ th pico eNB. B is the set of candidate sites for pico eNB.

(2)

$$
p_{k, l}, p_{m, q} \geq 0
$$

(3) The OI for the macro and pico eNB should be less than the OI threshold:

$$
\mathrm{OI}^{\text {macro eNB }}, \mathrm{OI}^{\text {pico eNB }}<I .
$$

3.2.1. Stage 1: Candidate Site Selection and Placement of Macro eNBs. The procedure for SSMPC based candidate site selection and placement of macro eNBs is shown in
Algorithm 6. The set of potential candidate sites for macro eNB, set of UEs covered by $k$ th macro eNB, TPB, and cost of macro eNB are given as the input to this stage. The identified candidate sites of macro eNB are the output from this stage. The SCI and PC are initialized to zero. SCI, OI, and DTI of each eNB candidate site are given as the input parameters for FIE and the corresponding output parameter SSI is obtained in steps (2) and (3), respectively. The candidate site with maximum SSI is identified in step (6). Based on this, SCI and PC are upgraded in steps (7) and (8), respectively. If the resultant $\mathrm{PC}$ is less than $\mathrm{TPB}$, the coordinates of identified candidate site with maximum SSI are stored in Sel_eNB. This is carried out in step (13). In step (15), the corresponding SSI is made to zero, so that, in step (6), the next highest SSI candidate site will be chosen for the next iteration. This procedure is rehashed until the condition in step (9) gets fulfilled. All the recognized macro eNB candidate sites with highest SSI are stored in Sel_eNB.

3.2.2. Stage 2: Power Resource Availability Check and Micro eNB Placement. The idea of SSMPC based power resource availability check in macro eNB and micro eNB placement is illustrated in Algorithm 7. The selected candidate sites of macro eNB from the first stage, noise power, SNR gap, target downlink information transmission rate, bandwidth supported per UE, UEs supported by $k$ th macro eNB, and maximum pico eNB capacity $(u)$ are the inputs to this stage. Identified macro and micro eNBs for placement and UEs that are not covered by any of the placed eNBs, SCI, and PC are the outputs from this stage.

In step (2), the transmit power required to serve all UEs covered by $k$ th eNB $\left(p_{k}\right)$ is initialized to zero. In step (4), the distance between $k$ th eNB and $l$ th UE is calculated. Based on the identified distance, path loss and transmit 


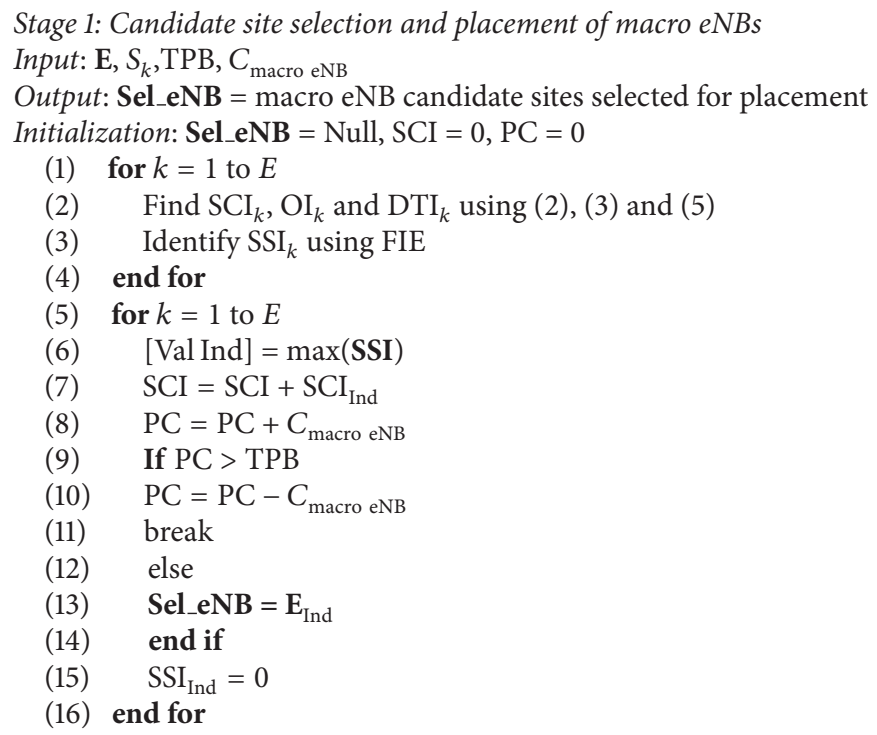

Algorithm 6: Candidate site selection and placement of macro eNBs for SSMPC approach.

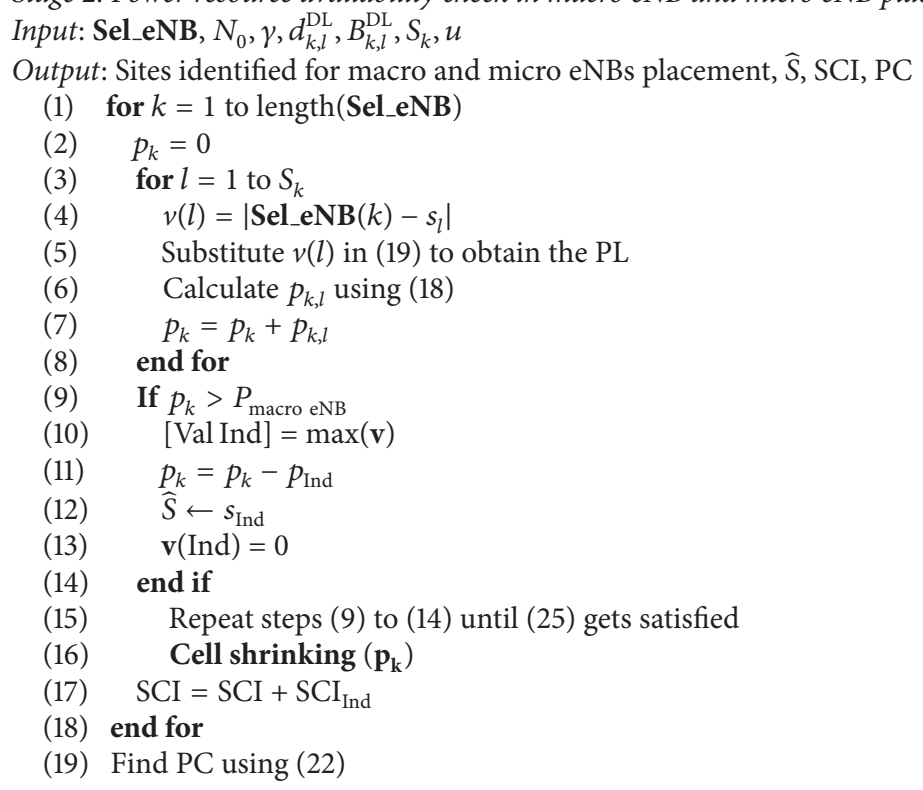

Algorithm 7: Power resource availability check in macro eNB and micro eNB placement for SSMPC approach.

power required to meet the target data rate are obtained using steps (5) and (6), respectively. This methodology is rehashed for all UEs covered under $k$ th eNB. The aggregate power required to bolster all these UEs is calculated in step (7). If the aggregate power required by $k$ th eNB exceeds $P_{\text {macro eNB }}$, then the outermost UEs are identified and expelled from present serving eNB one by one, until the power constraint in (25) gets fulfilled. This is done between steps (9) and (14). The expelled UEs are stored in $\widehat{S}$ and their related distances are made to zero in steps (12) and (13), respectively. This procedure is rehashed for all recognized macro eNBs until the condition in (25) gets fulfilled. When all the recognized macro eNBs fulfill the power constraint, the idea of cell shrinking is carried out, which is already briefed in Algorithm 3.

3.2.3. Stage 3: Candidate Site Selection and Placement of Pico $e N B s$. The procedure for SSMPC based candidate site selection and placement of pico eNBs is outlined in Algorithm 8. 
Stage 3: Candidate site selection and placement of pico eNBs

Input: SCI, PC, B, TPB, $\widehat{S}$

Output: Sel_pico = pico eNB candidate sites selected for placement

Initialization: Sel_pico $=$ Null

(1) for $m=1$ to $B$

(2) Calculate $\mathrm{SCI}_{m}, \mathrm{OI}_{m}$ and DTI $m$ using (2), (3) and (5)

(3) Identify $\mathrm{SSI}_{m}$ using FIE

(4) end for

(5) for $m=1$ to $B$

(6) $[$ Val Ind $]=\max ($ SSI $)$

(7) $\quad \mathrm{SCI}=\mathrm{SCI}+\mathrm{SCI}_{\text {Ind }}$

(8) $\mathrm{PC}=\mathrm{PC}+C_{\text {pico eNB }}$

(9) $\quad$ If $\mathrm{PC}>\mathrm{TPB}$

(10) $\mathrm{PC}=\mathrm{PC}-C_{\text {pico eNB }}$

(11) break

(12) else

(13) Sel_pico $=\mathbf{B}_{\text {Ind }}$

(14) end if

(15) SSI $_{\text {Ind }}=0$

(16) end for

Algorithm 8: Candidate site selection and placement of pico eNBs.

The SCI is upgraded after the completion of second stage and given as one of the inputs for stage 3 . The PC, set of candidate sites for pico eNB placement, TPB, and set of UEs that are not covered by any of the placed eNBs are also given as the input to this stage. The recognized candidate sites of pico eNB are the output from this stage. SCI, OI, and DTI of each pico eNB candidate site are given as the input parameters for FIE and the corresponding output parameter SSI is obtained in steps (2) and (3), respectively. The candidate site with maximum SSI is recognized in step (6). Based on this, SCI and PC are upgraded in steps (7) and (8), respectively. If the resultant $\mathrm{PC}$ is less than TPB, the coordinates of identified candidate site with maximum SSI are stored in Sel_pico in step (13). In step (15), the corresponding SSI is made to zero, so that, in step (6), the next highest SSI candidate site will be chosen for the next iteration. This procedure is rehashed until the condition in step (9) gets fulfilled. All the recognized pico eNB candidate sites with highest SSI are stored in Sel_pico.

3.2.4. Stage 4: Power Resource Availability Check for Pico eNBs and Pico eNB Selection. The idea of SSMPC based power resource availability check for placed pico eNBs and pico eNB selection to satisfy UEs service demand is outlined in Algorithm 9. The selected candidate sites of pico eNBs from the third stage, noise power, SNR gap, expected downlink information transmission rate $\left(d_{m, q}^{\mathrm{DL}}\right)$, bandwidth allocated per UE by $m$ th pico eNB $\left(B_{m, q}^{\mathrm{DL}}\right)$, and UEs supported by $m$ th pico eNB $\left(S_{m}\right)$ are the inputs to this stage. The pico eNBs selected to offer requested service demand are the outputs from this stage. In step (2), the transmit power required to serve all UEs covered by $m$ th pico eNB $\left(p_{m}\right)$ is initialized to zero. In step (4), the distance between $m$ th pico eNB and $q$ th $\mathrm{UE}$ is calculated. Based on the identified distance, path loss and transmit power required to meet the target data rate are obtained using steps (5) and (6), respectively. The transmit power required is obtained in step (6) using (16)-(18). This methodology is rehashed for all UEs covered under $m$ th pico eNB. The aggregate power required to bolster all these UEs is calculated in step (7).

If the aggregate power required by $m$ th pico eNB exceeds $P_{\text {pico eNB }}$, then the outermost UEs are identified and expelled from present serving pico eNB one by one, until the power constraint in (26) gets fulfilled. This process is repeated for all the selected pico eNBs. When all the selected pico eNBs satisfy the power constraint, the idea of pico eNB selection is carried out in step (12). The services of UEs, which are discarded by the current serving pico eNB, are handed over to the neighboring pico eNB based on the availability of the radio resources. This process minimizes the link traffic overloading and packet queuing delay.

The computational complexity of the proposed SSMSC approach is explained here. In stage 1, SCI, OI, and DTI are given as the input for the FIE. The SCI computation needs 1 multiplication. OI computation requires 24 multiplications, 14 additions, and 4 times' LUT access and the DTI computation needs 1 addition, 1 multiplication, and 1 comparison. SSI is calculated by the center of gravity method [25] using

$$
\mathrm{SSI}=\frac{\sum_{i=1}^{t} \psi_{D}\left(d_{i}\right) \cdot d_{i}}{\sum_{i=1}^{t} \psi_{D}\left(d_{i}\right)},
$$

where $t$ is the number of samples used to compute SSI. 


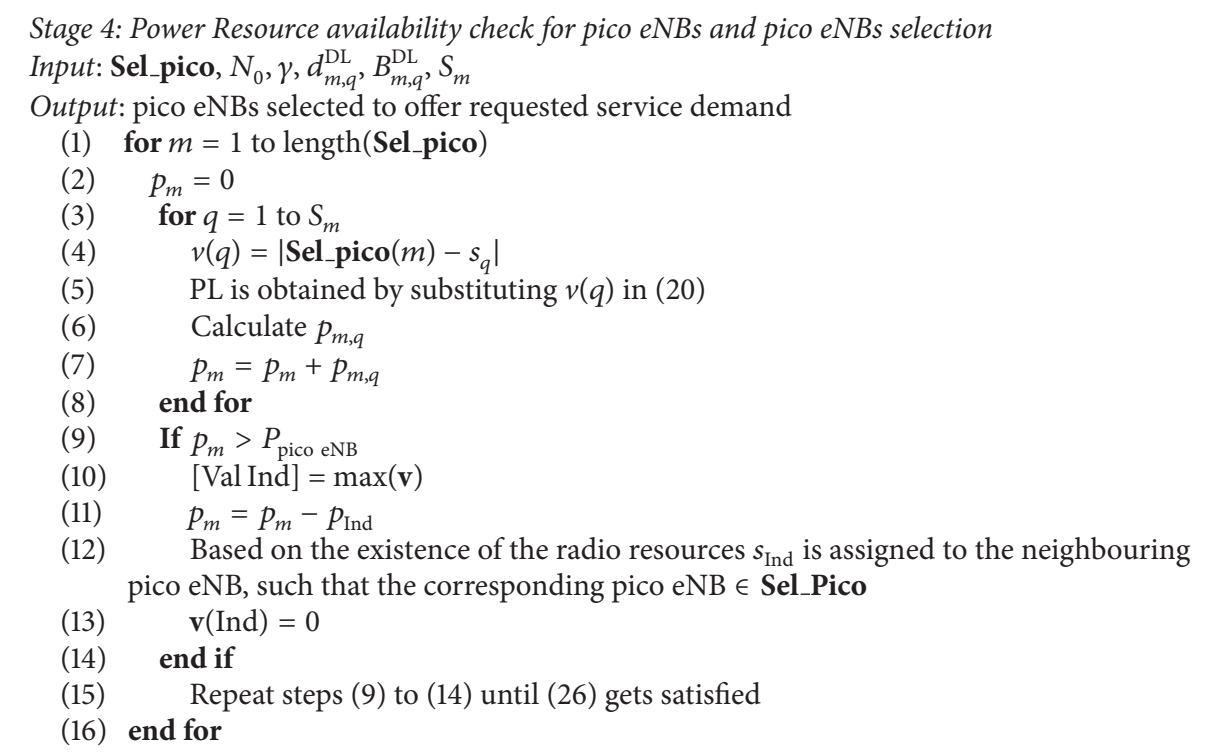

Algorithm 9: Power resource availability check for pico eNBs and pico eNBs selection.

The SSI computation in step (3) requires $2(t-1)$ additions and $(t+1)$ multiplications. Steps (1) to (4) are repeated for $\mathbf{E}$ candidate sites of eNB. The maximum SSI can be obtained using step (6), which needs $\mathbf{E}(\mathbf{E} \log \mathbf{E})$ comparisons. The SCI upgradation in step (7) needs 1 addition. Steps (5) to (14) are repeated for $\mathbf{E}$ candidate sites of eNB. Thus, stage 1 requires $\mathbf{E}(2 t+14)$ additions, $\mathbf{E}(t+27)$ multiplications, $\mathbf{E}(2+$ E $\log \mathbf{E})$ comparisons, and $4 \mathbf{E}$ times' LUT access, respectively. In stage 2 , the distance between every Sel_eNB and all the UEs covered within the $k$ th eNB is identified in step (4). This computation needs 3 multiplications, 5 additions, and 2 times' LUT access. In step (5), the PL calculation needs 1 addition, 1 multiplication, and 1-time LUT access. $p_{k, l}$ calculation in step (6) needs 1 addition, 1 multiplication, and 1-time LUT access. $p_{k}$ update in step (7) requires 1 addition. Steps (3) to (8) are iterated for $S_{k}$ number of times. Step (9) needs 1 comparison. The outermost UEs are identified using step (10), which needs $S_{k} \log S_{k}$ comparisons. In step (11), $p_{k}$ is updated which needs 1 addition. Here, steps (9) to (14) are repeated for $L$ number of times. The process of cell shrinking is carried out in step (16). This process needs $\left(u S_{k} \log S_{k}+2\right)$ comparisons and $(u+1)$ additions. The steps used in stage 2 are iterated for Sel_eNB number of times. Hence, the computation of stage 2 requires Sel_eNB $*\left(8 S_{k}+L+u+1\right)$ additions, Sel_eNB $* 5 S_{k}$ multiplications, Sel_eNB $*\left(L\left(S_{k} \log S_{k}+1\right)+\right.$ $\left.\left(u S_{k} \log S_{k}+2\right)\right)$ comparisons, and Sel_eNB $* 4 S_{k}$ times' LUT access, respectively. The computation of stages 3 and 4 is almost similar to that of stages 1 and 2 . The number of additions, multiplications, comparisons, and LUT access required by SSMSC approach is listed in Table 2. $g$ used in stage 4 represents the number of iterations required by steps (9) to (14) to fulfill the condition in (13). In a similar way, the computational complexity of SSMPC approach can be calculated, which is also listed in Table 3. $e$ used in stages 1 and 3 of Table 3 represents the number of iterations required between steps (9) and (14).

The complexity comparison of different HetNet/MHR placement approaches is listed in Table 4 . In Table $4, x$ is the number of identified clusters, $U_{s}$ is the number of UEs uncovered, and $N$ is the number of iterations needed to compute final mean point (MP). These variables are used to represent the computational complexity of uniform clustering approach. In ACRD approach, $M, F, C$, and $J$ represent the number of tiers in the eNB coverage, the number of RS types, the number of placement combinations, and the number of parameters weights, respectively. From Table 4, it is clear that the complexity of the uniform clustering approach is larger than the fuzzy logic based MHR placement approach. The conventional EDMC approach utilizes more complex and iterative MAHC, weighted $K$-means, and MGDC algorithms to identify the placement locations. This builds the overall computational complexity. Our proposed approaches use fuzzy logic for eNB and RS placement. Since most of the fuzzy operations are logical, the computational complexity of these approaches is less when compared to other HetNet placement approaches. Even though the fuzzy logic based MHR placement approach appears less complex than our proposed approaches, it lags in the SCI, PC, power proportion, and aggregate power requirement performances.

\section{Simulations and Discussions}

The performance of the proposed approaches is validated by the system level simulations using Matlab 2015a tool. The parameters and assumptions considered for the simulation study are listed in Table 5. The simulation is rehashed for 100 distinctive UE distributions and the average values of SCI, 
TABLE 2: Stage-wise complexity analysis of SSMSC approach.

\begin{tabular}{lllll}
\hline Stage & Number of additions & $\begin{array}{l}\text { Number of } \\
\text { multiplications }\end{array}$ & Number of comparisons & $\begin{array}{l}\text { Number of times Look } \\
\text { Up Table (LUT) is } \\
\text { accessed }\end{array}$ \\
\hline 1 & $E(2 t+14)$ & $E(t+27)$ & $E(2+E \log E)$ & $4 E$ \\
2 & Sel_eNB $*\left(8 S_{k}+L+u+1\right)$ & Sel_eNB $* 5 S_{k}$ & Sel_eNB $*\left(L\left(S_{k} \log S_{k}+1\right)+\left(u S_{k} \log S_{k}+2\right)\right)$ & Sel_eNB $* 4 S_{k}$ \\
3 & $R(2 t+14)$ & $R(t+27)$ & $R(2+R \log R)$ & $4 R$ \\
4 & Sel_RS $*\left(8 S_{n}+2 g\right)$ & Sel_RS $* 5 S_{n}$ & Sel_RS $* g\left(S_{n} \log S_{n}+2\right)$ & Sel_RS $* 4 S_{n}$ \\
\hline
\end{tabular}

TABLE 3: Stage-wise complexity analysis of SSMPC approach.

\begin{tabular}{lllll}
\hline Stage & Number of additions & $\begin{array}{l}\text { Number of } \\
\text { multiplications }\end{array}$ & Number of comparisons & $\begin{array}{l}\text { Number of times LUT is } \\
\text { accessed }\end{array}$ \\
\hline 1 & $E(2 t+15)+e$ & $E(t+27)$ & $E(2+E \log E)$ & $4 E$ \\
2 & Sel_eNB $*\left(8 S_{k}+L+u+2\right)+1$ & Sel_eNB $* 5 S_{k}+E+B$ & $\begin{array}{l}\text { Sel_eNB } *\left(L\left(S_{k} \log S_{k}+1\right)+\right. \\
\left.\left(u S_{k} \log S_{k}+2\right)\right)+1\end{array}$ & Sel_eNB $* 4 S_{k}$ \\
3 & $B(2 t+15)+e$ & $B(t+27)$ & $B(2+B \log B)$ & $4 B$ \\
4 & Sel_pico $*\left(8 S_{m}+2 g\right)$ & Sel_pico $* 5 S_{m}$ & Sel_pico $* g\left(S_{m} \log S_{m}+2\right)$ & Sel_pico $* 4 S_{m}$ \\
\hline
\end{tabular}

PC, and an aggregate power required by all placed serving nodes and power proportion are displayed in this work.

\section{Assumptions}

(i) The candidate placement sites for diverse eNBs and $\mathrm{RS}$ are randomly selected within the geographic area to be covered.

(ii) The proposed approaches are tested only for downlink traffic demands.

The stage-by-stage execution of one of the sample simulation scenarios of the proposed SSMSC approach is shown in Figures 11-14, respectively. In the first stage of placement, 7 macro eNB candidate sites are chosen out of 16 candidate sites in light of the fuzzy logic. This is shown in Figure 11. During the second stage, power resource availability check is carried out for every placed macro eNB. Based on the availability of the radio resources and the number of covered clients, 2 of the macro eNBs are made to micro eNBs by cell shrinking. Due to this, some of the UEs become uncovered. The services of these UEs are handed over to the neighboring placed macro eNBs based on the existence of radio resources. A sample scenario corresponding to the second stage is shown in Figure 12. The services of some of the uncovered UEs in the overlapping area are handed over to the neighboring macro eNB, which is also highlighted by blue color lines in Figure 12. RSs are utilized to maximize the SCI and to support the UEs in the dead areas. Some of the UEs in the coverage region of macro or micro eNBs may not get service due to the shortage of radio resources. These UEs are also served by the RSs. For the given scenario, the proposed SSMSC approach selects 7 candidate sites of RSs out of 18 . The sample simulation scenario corresponding to stage 3 is illustrated in Figure 13. As shown in Table 1, the number of clients supported by the $\mathrm{RS}$ is much lower than macro eNB. Deficient resources may prompt link traffic overloading in RSs. During the fourth stage, some of the UEs in RS overlapping area are handed over to the neighbouring RSs based on the accessibility of radio resources. This is highlighted with red colour lines. The sample simulation scenario for stage 4 is shown in Figure 14. The RS selection carried out in stage 4 maximizes the average throughput per user and minimizes the packet queuing delay. Through simulations, it is validated that our proposed SSMSC approach offers coverage more than the expected for every scenario considered. From the final placement sites, we can conclude that the OI for all the placed serving nodes is lower than the interference threshold considered.

The aggregate PC required by different HetNet/MHR placement approaches is compared in Figure 15. It is noted that the PC increases with the UE density. To bolster the increasing number of UEs and their traffic demands, the network operators have to increase the serving node density. This increases the PC and cost per bit. To meet the target SCI, MCD approach always considers the placement of macro eNBs irrespective of the number of uncovered UEs. This builds the PC. Since this approach does not utilize small cells, the PC of this approach is always higher than the other approaches. The PC of ATD and TKD approaches are almost the same. Both of these approaches utilize MAHC algorithm to decide the required number of macro eNBs. This may result in the same number of macro eNBs for both of these approaches. Since ATD approach does not utilize pico eNBs, it needs marginally more number of micro eNBs than TKD approach to meet the target SCI. The MHR placement approaches like ACRD, uniform clustering, and fuzzy require approximately the same $\mathrm{PC}$. In these approaches, the placement of eNB and RS is alone considered. ACRD approach considers maximum number of RS combinations and chooses more number of RSs than uniform and fuzzy based placement approaches to meet the target SCI. In [32], it 
TABLE 4: Complexity comparison of different HetNet/MHR placement approaches.

\begin{tabular}{|c|c|c|c|c|}
\hline Approaches & Number of additions & Number of multiplications & Number of comparisons & $\begin{array}{l}\text { Number of times LUT is } \\
\text { accessed }\end{array}$ \\
\hline $\begin{array}{l}\text { Uniform } \\
\text { clustering }\end{array}$ & $\begin{array}{l}13 T-2+4 x+x N(T-1)+ \\
5 x E+E R\left(U_{s}-1\right)+E R(E+R- \\
1)+(E+R)+2(E+R)(T-1)+ \\
E(x-1)+E(E+R-1)+5 R\end{array}$ & $\begin{array}{l}8 x+x N+3 x E+3 E R+ \\
E R(E+R)+2(E+R)+ \\
E(E+R)+19 T+14 R+2\end{array}$ & $\begin{array}{l}x(E \log E)+E R(R \log R)+ \\
E R+2 E+R\end{array}$ & $5 T+2 x+2 x E+3 R$ \\
\hline Fuzzy & $\begin{array}{l}E(2 t-1)+E R\left(U_{s}-1\right)+ \\
E R(E+R-1)+2 R(T-1)+ \\
6 R+(E-1)+E(E+R-1)+6 T\end{array}$ & $\begin{array}{l}E(t+3)+3 E R+E R(E+R)+ \\
E(E+R)+7 R+7 T+1\end{array}$ & $2 E+E R(R \log R+1)$ & $3(T+R)$ \\
\hline ACRD & $\begin{array}{l}4 M(F-1)+5(M-1)+C M F+ \\
M(F+1)+4(J-1)+3 C\end{array}$ & $\begin{array}{l}5 M F+3(M+1)+2 M+ \\
C M F+C M(2 F+2)+4 C+17\end{array}$ & $3 C$ & - \\
\hline EDMC & $\begin{array}{l}7 T+4 x+x N(T-1)+ \\
\text { Sel_eNB } *\left(8 S_{k}+L+u+1\right)+ \\
\text { Sel_pico } *\left(8 S_{m}+g\right)\end{array}$ & $\begin{array}{l}3 T+8 x+x N+7+\text { Sel_eNB } * \\
\left(5 S_{k}\right)+\text { Sel_pico } *\left(5 S_{m}\right)\end{array}$ & $\begin{array}{l}T(T-1)+\text { Sel_eNB } * \\
\left(L\left(S_{k} \log S_{k}+1\right)+\left(u S_{k} \log S_{k}+\right.\right. \\
2))+ \text { Sel_pico } *\left(g\left(S_{m} \log S_{m}+\right.\right. \\
1))\end{array}$ & $\begin{array}{l}2 T+2 x+\text { Sel_eNB } * \\
\left(4 S_{k}\right)+\text { Sel_pico } *\left(4 S_{m}\right)\end{array}$ \\
\hline SSMSC & $\begin{array}{l}E(2 t+14)+\text { Sel_eNB } * \\
\left(8 S_{k}+L+u+1\right)+R(2 t+ \\
14)+ \text { Sel_RS } *\left(8 S_{n}+2 g\right)\end{array}$ & $\begin{array}{l}E(t+27)+\text { Sel_eNB } * 5 S_{k}+ \\
R(t+27)+\text { Sel_RS } * 5 S_{n}\end{array}$ & $\begin{array}{l}E(2+E \log E)+\text { Sel_eNB } * \\
\left(L\left(S_{k} \log S_{k}+1\right)+\left(u S_{k} \log S_{k}+\right.\right. \\
2))+R(2+R \log R)+\text { Sel_RS } * \\
g\left(S_{n} \log S_{n}+2\right)\end{array}$ & $\begin{array}{l}4 E+\text { Sel_eNB } * 4 S_{k}+4 R+ \\
\text { Sel_RS } * 4 S_{n}\end{array}$ \\
\hline SSMPC & $\begin{array}{l}E(2 t+15)+e+\text { Sel_eNB } * \\
\left(8 S_{k}+L+u+2\right)+1+B(2 t+ \\
15)+e+\text { Sel_pico } *\left(8 S_{m}+2 g\right)\end{array}$ & $\begin{array}{l}E(t+27)+\text { Sel_eNB } * 5 S_{k}+E+ \\
B+B(t+27)+\text { Sel_pico } * 5 S_{m}\end{array}$ & $\begin{array}{l}E(2+E \log E)+\text { Sel_eNB } * \\
\left(L\left(S_{k} \log S_{k}+1\right)+\left(u S_{k} \log S_{k}+\right.\right. \\
2))+1+B(2+B \log B)+ \\
\text { Sel_pico } * g\left(S_{m} \log S_{m}+2\right)\end{array}$ & $\begin{array}{l}4 E+\text { Sel_eNB } * 4 S_{k}+4 B+ \\
\text { Sel_pico } * 4 S_{m}\end{array}$ \\
\hline
\end{tabular}

has been proved that the fuzzy approach requires less number of RSs than the uniform clustering approach. This results in slightly less PC than uniform clustering approach. Since these MHR placement approaches do not efficiently utilize the LTE small cells, they do not show a significant gain than ATD and TKD approaches. In EDMC approach, macro, micro, and pico eNBs are utilized to bolster the traffic demands of the service requesting UEs. When there exist less number of UEs in a macrocell, the idea of range adjustment is executed. Based on this, some of the macrocells are made to microcells by adjusting the transmitted signal power. To support the uncovered UEs, pico eNBs are placed based on the MGDC algorithm. This EDMC approach offloads the uneven traffic demands in the hotspots to small cells. The usage of more number of micro- and picocells reduces the PC. The proposed SSMSC approach decides the placement locations of macro eNB, micro eNB, and RS by considering the traffic demands and the spatial distribution of UEs. This approach utilizes fuzzy logic for both macro eNB and RS placement. The usage of fuzzy logic minimizes the unwanted placements. The idea of cell shrinking is utilized to decide the micro eNB locations. Our proposed SSMSC approach motivates the placement of more number of small cells than the macrocell. Since an RS can cover more number of UEs than the micro and pico eNBs, our proposed approach requires less number of RSs than the other MHR placement approaches to meet the target SCI. This in turn minimizes the PC and cost per bit than the other conventional placement approaches. The effective SSMSC approach reduces the PC approximately $7 \%$ compared to EDMC approach.

In Figure 16, the aggregate powers required by all serving nodes (in W) are compared between different HetNet/MHR placement approaches for the target SCI of $90 \%$. As indicated in Table 5, the traffic demand of each UE is varied between 0.1 and $1 \mathrm{Mbps}$. To meet the target information rate, eNB and RS have to distribute distinctive transmit power levels to each UE taking into account the noise, interference, path loss, and fading channel conditions. Increment in the traffic demand will likewise build the transmit power level, which likewise expands the aggregate power required by all serving nodes. MCD approach utilizes large transmit power to cover more number of UEs. To cover the cell edge users, the placed macro eNBs have to increase the transmit power levels, which also increases the aggregate transmission power. Since this approach always place macro eNBs, the aggregate transmission power required to support the clients demand is always higher than the other approaches. In ATD approach, the uncovered UEs are supported by micro eNBs. This will lessen the aggregate power requirement of ATD approach compared to MCD approach. To support the uncovered UEs, pico eNBs are used along with micro eNBs, which makes TKD approach conserve more power than ATD approach. ACRD, uniform clustering, and fuzzy approaches utilize RSs to support the uncovered UEs. Since an RS can cover more number of UEs than micro and pico eNBs, the aggregate transmission power required by these approaches is always less than MCD, ATD, and TKD approaches. In EDMC approach, the range adjustment based micro eNB and MGDC based pico eNB placement methodologies are utilized, which reduces the aggregate power consumption. The usage of more number of micro and pico eNBs to bolster the uncovered UEs with nonuniform traffic demands lessens the transmission power. Our proposed SSMSC approach places blend of macro eNB, micro eNB, and RS adequately, which makes serving 
TABLE 5: Simulation settings.

\begin{tabular}{lc}
\hline \multicolumn{1}{c}{ Serving node $(e N B / R S)$ settings } \\
\hline Carrier frequency $(\mathrm{GHz})$ & 2.14 [29] \\
\hline System bandwidth $(\mathrm{MHz})$ & Micro eNB: 100 \\
& Pico eNB: 30 \\
RS: 50 \\
\hline & Macro eNB: 32 \\
PC (units) & Micro eNB: 3.5 \\
& Pico eNB: 1 \\
& RS: 4.5 \\
Total power allocated to & Macro eNB: 40 \\
serving nodes (W) & Micro eNB: 2 \\
& Pico eNB: 0.25 \\
& RS: 4 \\
\hline & Macro eNB: 3 \\
Coverage radius $(k m)$ & Micro eNB: 1 \\
& Pico eNB: 0.25 \\
\hline Traffic model & RS: 1.125 \\
\hline Interference threshold or & Full buffer [30] \\
OI (\%) & $<25 \%$ \\
\hline
\end{tabular}

\begin{tabular}{lc}
\hline & Network layout parameters \\
\hline $\begin{array}{l}\text { Scenario } \\
\text { Size of the geographic area }\end{array}$ & Large group \\
\hline $\begin{array}{l}\text { Network nodes } \\
\text { macro eNB, micro eNB, pico eNB, } \\
\text { RS, and UE }\end{array}$ \\
$\begin{array}{lc}\text { Number of macro eNB } \\
\text { candidate sites }\end{array}$ \\
$\begin{array}{l}\text { Number of RS candidate } \\
\text { sites }\end{array}$ & 16 \\
\hline $\begin{array}{l}\text { Number of pico eNB } \\
\text { candidate sites }\end{array}$ & 100 \\
\hline
\end{tabular}

\begin{tabular}{|c|c|}
\hline \multicolumn{2}{|c|}{ Channel parameters } \\
\hline Path loss model & Log-distance path loss model [4] \\
\hline Small scale fading type & slow fading \\
\hline Fading model & Jakes Rayleigh [31] \\
\hline Shadowing model & Log normal shadowing [4] \\
\hline Shadowing factor (dB) & $\begin{array}{c}\text { macro eNB: } 10 \mathrm{~dB} \\
\text { micro eNB: } 6 \mathrm{~dB} \\
\text { pico eNB: } 6 \mathrm{~dB} \\
\text { RS: } 6 \mathrm{~dB}\end{array}$ \\
\hline $\begin{array}{l}\text { Additive white Gaussian } \\
\text { noise }(\mathrm{AWGN}) \text { power } \\
(\mathrm{dBm} / \mathrm{Hz})\end{array}$ & $-174[4]$ \\
\hline \multicolumn{2}{|c|}{ UE settings } \\
\hline UE density & 600 to 1000 \\
\hline Spatial distribution & $\begin{array}{c}\text { Nonhomogeneous Poisson and } \\
\text { uniform process [1] }\end{array}$ \\
\hline Velocity of UE (kmph) & $\leq 5$ \\
\hline Noise figure $(\mathrm{dB})$ & 9 \\
\hline $\begin{array}{l}\text { Downlink traffic demand } \\
\text { (Mbps) }\end{array}$ & 0.1 to $1 \mathrm{Mbps}$ \\
\hline
\end{tabular}

TABLE 5: Continued.

\begin{tabular}{ll}
\hline \multicolumn{2}{c}{ Other parameters } \\
\hline Expected bit error rate & $10^{-6}$ \\
\hline SNR gap & 7.63 \\
\hline Target SCI & $90 \%$ \\
\hline TPB & 200 units
\end{tabular}

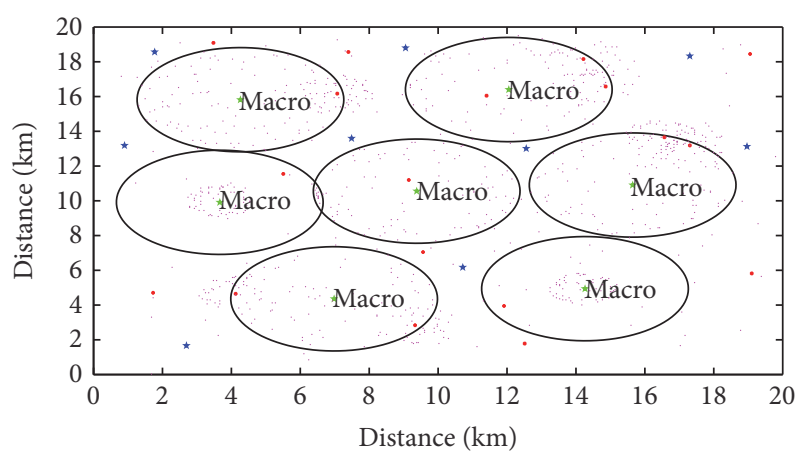

FIGURE 11: Sample simulation scenario for stage 1 of SSMSC approach.

nodes closer to UEs. In this approach, the underutilized macrocells are made to microcells, which also minimize the aggregate power consumption. From Figure 16, it is clear that the power consumption performance of our proposed approach is superior to the other conventional approaches. To meet the target information rate of $1 \mathrm{Mbps}$, the aggregate power required by all the placed serving nodes of SSMSC approach is $154 \mathrm{~W}$. From Figure 14, it is noted that the aggregate power constraint of proposed placement approach is $232 \mathrm{~W}$. Thus, our proposed approach satisfies the power constraint.

Figure 17 shows power proportion comparison between various HetNet/MHR placement approaches for the target SCI of $90 \%$. The power proportion is the measure of proportion of power required by macro eNB, micro eNB, pico eNB, and RS to accomplish the target traffic demand. Since MCD approach places only macro eNBs, the power consumed by macro eNB is $100 \%$, whereas for other kind of small cells it is zero. Since ATD and TKD approaches utilize MAHC algorithm for macro eNB placement, the powers consumed by macro eNBs of these approaches are almost the same. In these two approaches, the power consumed by macro eNB is approximately $97 \%$. In ATD approach, the remaining $3 \%$ power is consumed by the placed micro eNBs. In TKD approach, the small cells like micro- and picocells consume the power of $1.9 \%$ and $1.1 \%$, respectively. The powers consumed by the placed RSs of ACRD, uniform clustering, and fuzzy approaches are $20.2 \%, 19.8 \%$, and $19.2 \%$, respectively. ACRD approach places slightly more number of RSs than the uniform clustering and fuzzy approaches to meet the target SCI. The power consumed by the small cells of EDMC and SSMSC approaches is $22.2 \%$ and $28.4 \%$, respectively. This verifiably demonstrates that our proposed SSMSC approach effectively uses LTE HetNet and MHR. 


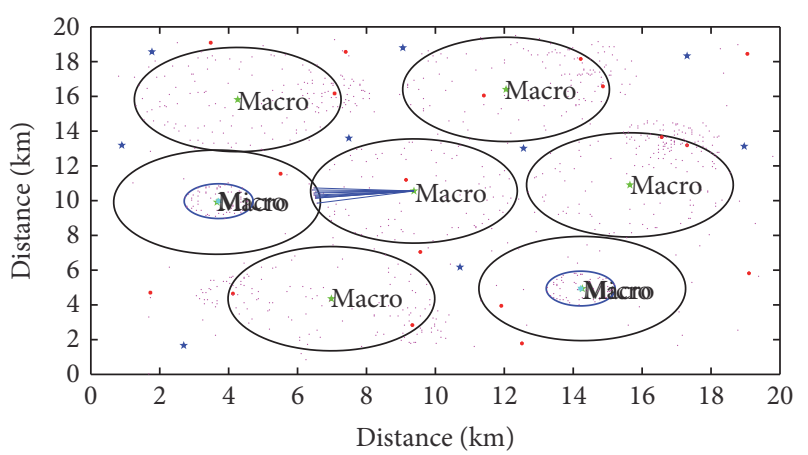

Figure 12: Sample simulation scenario for stage 2 of SSMSC approach.

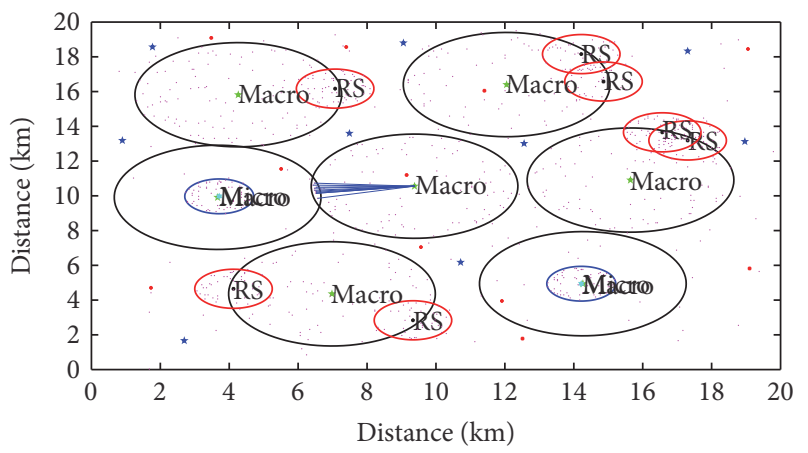

FIGURE 13: Sample simulation scenario for stage 3 of SSMSC approach.

Hence, this approach efficiently solves the traffic demand and coverage problems experienced by the UEs in the hotspots and dead areas.

The SINR of $l$ th UE corresponding to $k$ th macro eNB can be obtained using [33-35]

$$
\operatorname{SINR}_{k, l}=\frac{p_{k, l}^{r}\left|h_{k, l}\right|^{2}}{N_{0}+\sum_{\substack{k^{\prime}=1 \\ k^{\prime} \neq k}}^{E} p_{k^{\prime}, l}^{r}\left|h_{k^{\prime}, l}\right|^{2}+\sum_{n=1}^{R} p_{n, l}^{r}\left|h_{n, l}\right|^{2}},
$$

where $k^{\prime}$ represents the neighboring macro eNBs, $p_{k^{\prime}, l}^{r}$ is the power received by the $l$ th UE from the $k^{\prime}$ th macro eNB, $\left|h_{k^{\prime}, l}\right|^{2}$ is the fading channel gain between the $k^{\prime}$ th macro eNB and $l$ th UE, $p_{n, l}^{r}$ is the power received by the $l$ th UE from $n$th RS, and $\left|h_{n, l}\right|^{2}$ is the fading channel gain between the $n$th RS and $l$ th UE.

The SINR of $r$ th UE corresponding to $n$th RS can be obtained using

$$
\operatorname{SINR}_{n, r}=\frac{p_{n, r}^{r}\left|h_{n, r}\right|^{2}}{N_{0}+\sum_{\substack{n^{\prime}=1 \\ n^{\prime} \neq n}}^{R} p_{n^{\prime}, r}^{r}\left|h_{n^{\prime}, r}\right|^{2}+\sum_{k=1}^{E} p_{k, r}^{r}\left|h_{k, r}\right|^{2}},
$$

where $p_{n, r}^{r}$ is the power received by the $r$ th UE from the $n$th RS, $\left|h_{n, r}\right|^{2}$ is the fading channel gain between the $n$th RS and $r$ th UE, $n^{\prime}$ represents the neighboring RSs, $p_{n^{\prime}, r}^{r}$ is the power

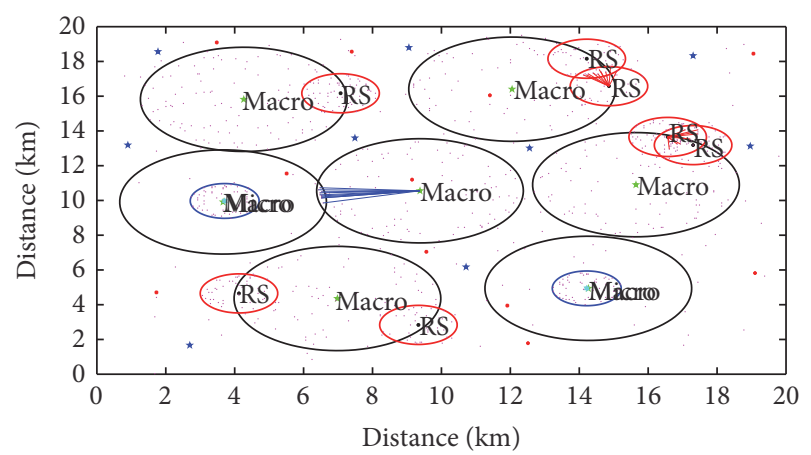

FIGURE 14: Sample simulation scenario for stage 4 of SSMSC approach.

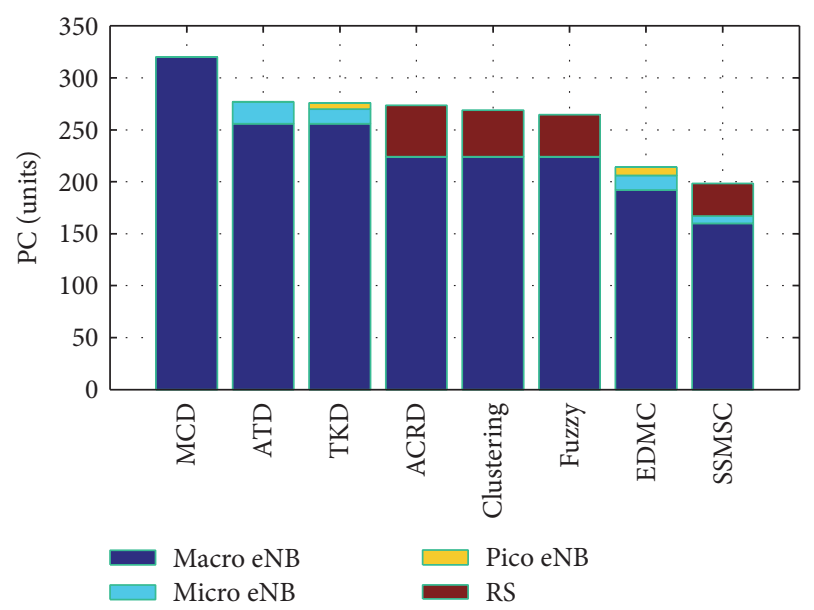

FIGURE 15: PC comparison between different HetNet/MHR placement approaches for the target SCI of $90 \%$.

received by the $r$ th UE from the $n^{\prime}$ th RS, $\left|h_{n^{\prime}, r}\right|^{2}$ is the fading channel gain between the $n^{\prime}$ th RS and $r$ th UE, $p_{k, r}^{r}$ is the power received by the $r$ th UE from the $k$ th macro eNB, and $\left|h_{k, r}\right|^{2}$ is the fading channel gain between the $k$ th macro eNB and $r$ th UE.

Figure 18 shows the user experiencing data rate comparison between various HetNet/MHR placement approaches for the target SCI of $90 \%$. This result is obtained by using the interference model proposed in LTE Rel-10 [33-35]. MCD approach places number of macro eNBs to achieve the target SCI. This increases the interference between the serving nodes, especially for the UEs on the cell edges. This reduces the SINR as well as the user experiencing data rate. Due to the inclusion of small cells, ATD, TKD, and EDMC approaches offer higher data rate than MCD approach. Due to the inclusion of RSs, ACRD, uniform clustering, and fuzzy based placement approaches offer higher data rate than the MCD approach. The fuzzy based approach achieves the expected SCI with less number of RSs than the uniform clustering and ACRD approach. This leads to reduced interference. Hence, the data rate experienced by the users of the fuzzy based placement approach is larger than ACRD and uniform clustering based approaches. But, all these approaches do 


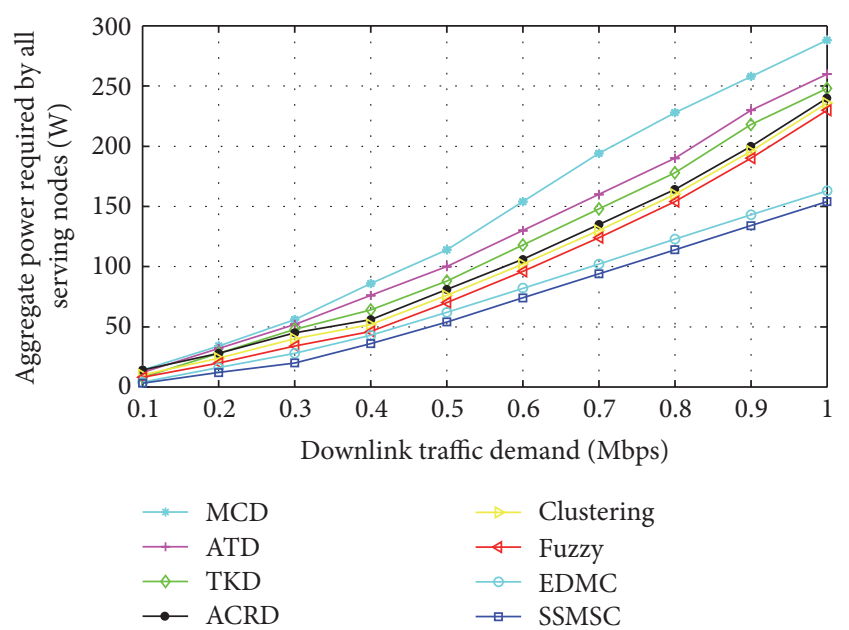

FIGURE 16: The aggregate power required by all serving nodes (in W) comparison between different HetNet/MHR placement approaches for the target SCI of $90 \%$.

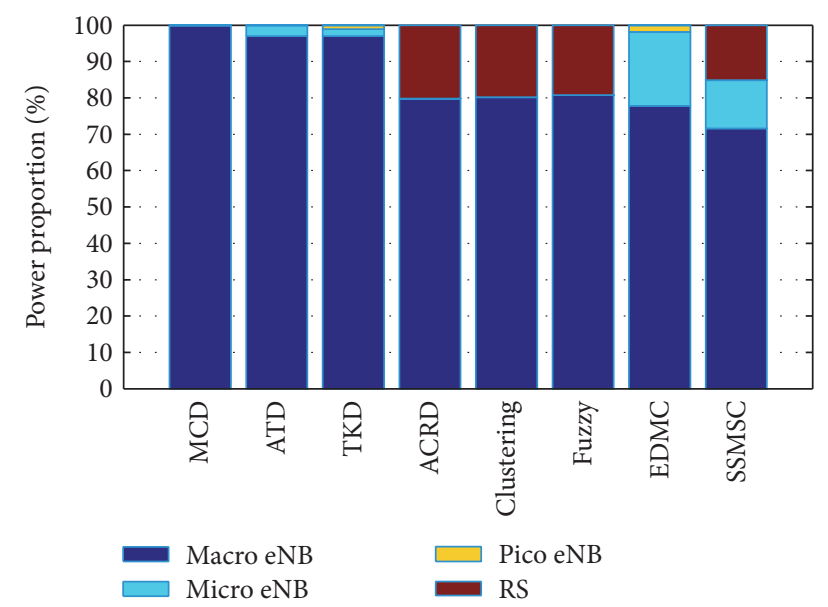

FIGURE 17: Power proportion comparison between different HetNet/MHR placement approaches for the target SCI of $90 \%$.

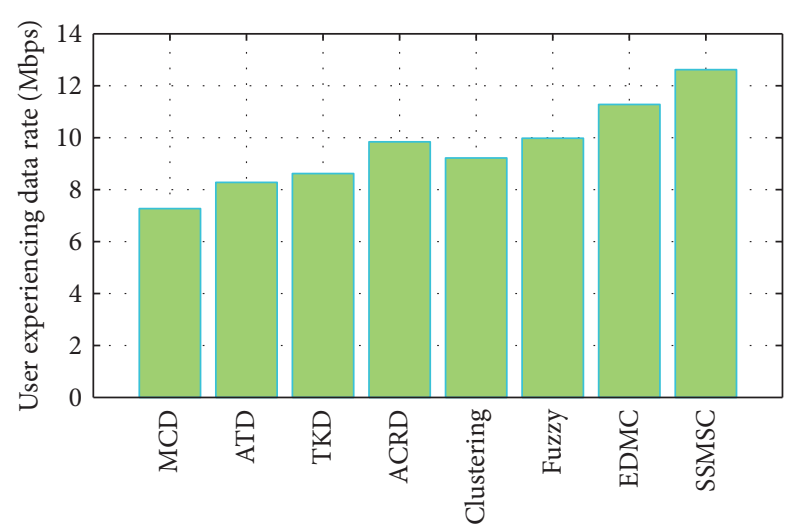

FIGURE 18: User experiencing data rate (Mbps) comparison between different HetNet/MHR placement approaches for the target SCI of $90 \%$. not consider the interference constraint. They allow more overlapping between the coverage areas of the placed serving nodes. This leads to large cotier and cross-tier interference and reduces the user experiencing data rate. From Figure 14, it is clear that the OI for all the placed serving nodes of SSMSC approach is lower than the considered interference threshold. SSMSC approach offers an average data rate of 12.62 Mbps, whereas the conventional EDMC approach offers $11.28 \mathrm{Mbps}$. The minimum interference nature of SSMSC approach increases the data rate approximately by $10 \%$ compared to EDMC approach.

The stage-by-stage execution of one of the sample simulation scenarios of the proposed SSMPC approach is shown in Figures 19-22, respectively. In the first stage of placement, 6 macro eNB candidate sites are chosen out of 16 candidate sites in light of the fuzzy logic. This is shown in Figure 19. During the second stage, power resource availability check is carried out for every placed macro eNB. Based on the availability of the radio resources and the number of covered users, 2 of the macro eNBs are made to micro eNBs by cell shrinking. Due to this, some of the UEs become uncovered. The services of these UEs are handed over to the neighboring placed macro eNBs based on the existence of radio resources. A sample scenario corresponding to the second stage is shown in Figure 20. The services of some of the uncovered UEs in the overlapping area are handed over to the neighboring macro eNB, which is also highlighted by blue color lines in Figure 20. Pico eNBs are utilized to maximize the SCI and to support the UEs in the dead areas and hotspots. Some of the UEs in the coverage region of macro or micro eNBs may not get service due to the shortage of radio resources. These UEs are also served by the pico eNBs. For the given scenario, the proposed SSMPC approach selects 65 candidate sites of pico eNBs out of 100 . The sample simulation scenario corresponding to stage 3 is illustrated in Figure 21. As shown in Table 1, the number of users supported by the pico eNB is much lower than macro eNB. Deficient resources may prompt link traffic overloading in pico eNBs. During the fourth stage, some of the UEs in pico eNB overlapping area are handed over to the neighbouring pico eNBs based on accessibility of radio resources. This is highlighted with black colour lines. In order to do this, LTE Rel-9 have introduced mobility load balancing (MLB) protocol, which exchanges information about load level and radio resource status between eNBs. The radio resource block status contains the information about the percentage of allocated physical resource blocks (PRB) and the percentage of available PRBs for load balancing. The zoomed sample simulation scenario for stage 4 is shown in Figure 22. The pico eNB selection carried out in stage 4 maximizes the system capacity and minimizes the packet queuing delay. From the final placement sites, we can conclude that the OI for all the placed serving nodes is lower than the interference threshold considered.

Figure 23 analyses the SCI offered by different HetNet/ MHR placement approaches for the given TPB of 200 units. MCD approach places 6 macro eNBs for the given budget. Since this approach dependably inclines toward just macro eNBs, it cannot serve the UEs in the dead areas and hotspots. Since ATD and TKD approaches incline towards small cells 


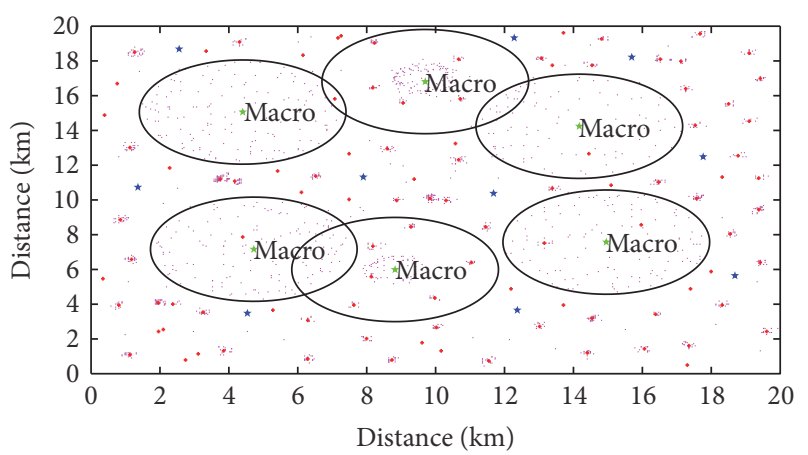

Figure 19: Sample simulation scenario for stage 1 of SSMPC approach.

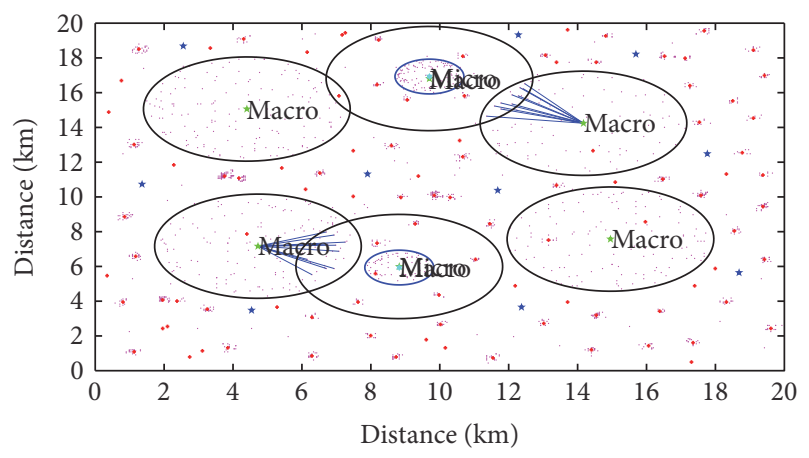

FIgURE 20: Sample simulation scenario for stage 2 of SSMPC approach.

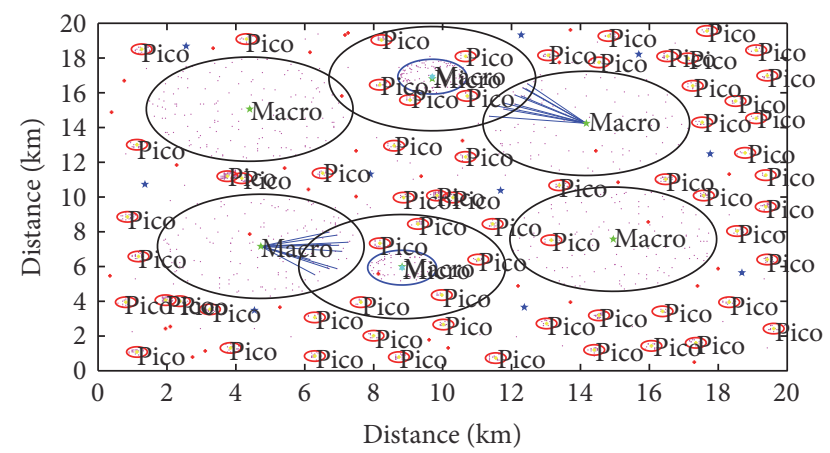

FIgURE 21: Sample simulation scenario for stage 3 of SSMPC approach.

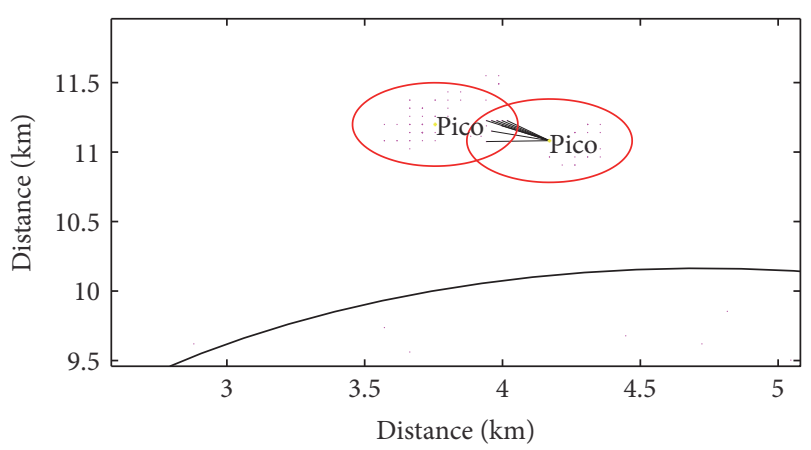

FIgURE 22: Sample simulation scenario for stage 4 of SSMPC approach.

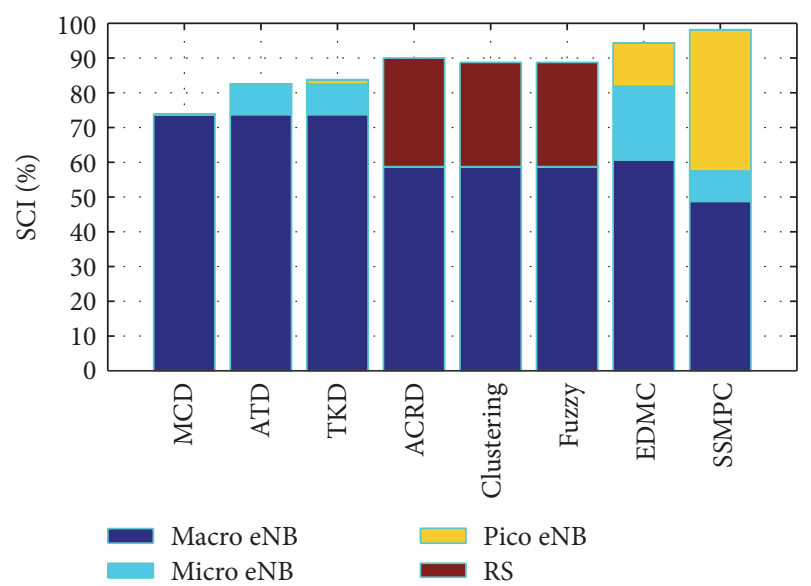

FIGURE 23: SCI (\%) comparison between different HetNet/MHR placement approaches for the TPB of 200 units.

alongside macro eNBs, they offer preferred SCI performance over MCD approach. ATD and TKD approaches utilize MAHC algorithm for macro eNB placement; they result in the same number of macro eNBs. Since TKD approach additionally places pico eNBs, the SCI performance of TKD approach is somewhat better than ATD approach. The MHR placement approaches like ACRD, uniform clustering, and fuzzy offer improved SCI performance compared to ATD and TKD approaches. This is mainly due to the larger coverage range of RS than the micro and pico eNBs. As in Table 1, the footprint and capacity of RS are much larger than pico eNB. Hence, these MHR placement approaches can accommodate more number of clients. EDMC approach effectively decides and places macro, micro, and pico eNBs. Because of the use of more number of small cells, the SCI performance of EDMC approach is higher than the other discussed approaches. MGDC approach utilized for pico eNB placement is developed to bolster maximum of two UEs. Because of the budget constraint, there is a limitation in terms of the number of pico eNBs, which thusly reduces the SCI. The proposed fourstage SSMPC approach efficiently identifies the quantity of eNBs and their heterogeneity. Because of the effectiveness of fuzzy based pico eNB placement, the SCI performance of our SSMPC approach is better than the alternate approaches considered. The proposed SSMPC approach offers 3.75\% improvement in SCI over the EDMC approach.

In Figure 24, the aggregate powers required by all serving eNBs (in W) are compared between different HetNet/MHR placement approaches for the TPB of 200 units. To fulfill the target budget constraint, the HetNet placement approaches like $\mathrm{MCD}, \mathrm{ATD}$, and TKD require the same number of macro eNBs. In any case, all the approaches other than MCD approach utilize small cells. In this way, the aggregate transmission power required by the placed serving nodes of MCD approach is lesser than ATD and TKD approaches. The aggregate transmission powers required by ACRD, uniform clustering, and fuzzy approaches are almost the same. EDMC approach requires less transmission power than the above approaches. This is because of the proficient use of small cells. 


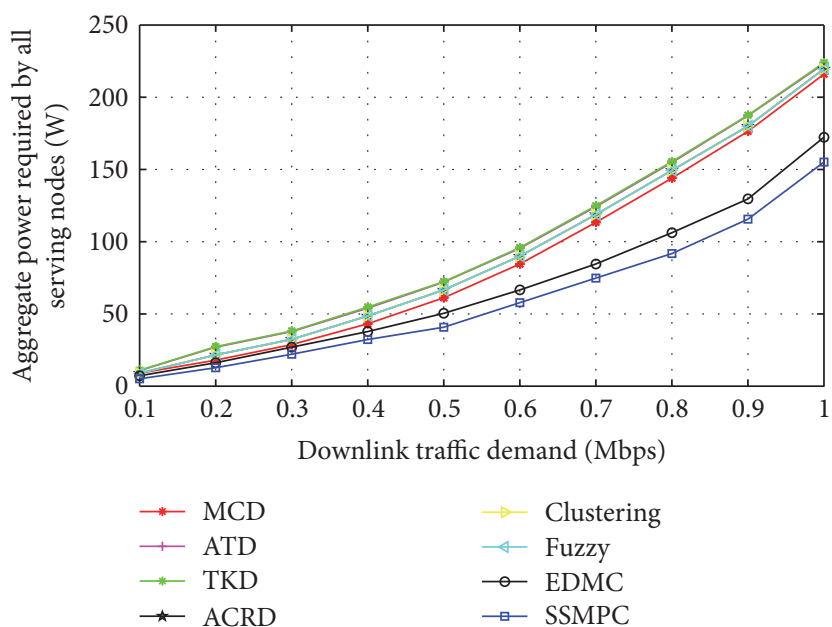

FIGURE 24: The aggregate power required by all serving nodes (in W) comparison between different HetNet/MHR placement approaches for the TPB of 200 units.

Our proposed SSMPC approach requires still lower aggregate power than the EDMC approach. This is mainly because of the accompanying reasons: The best possible eNB choice and placement convey UEs nearer to the serving eNBs. The decrease in transmission distance lessens the transmission power requirement. This approach likewise productively offloads the part of the traffic demand to small cells. The idea of cell shrinking used in stage 2 also minimizes the unnecessary wastage of transmission power. From Figure 24, it is clear that, to accomplish the target information rate of $1 \mathrm{Mbps}$, the aggregate power required by all the serving nodes of SSMPC approach is $155.10 \mathrm{~W}$. From Figure 21, it is noticed that the aggregate power constraint is $180.25 \mathrm{~W}$. Thus the proposed approach satisfies the power constraint given in (25) and (26). To achieve the target information rate of $1 \mathrm{Mbps}$, the SSMPC approach shows an improvement of approximately $10 \%$ over the EDMC approach.

Figure 25 shows power proportion comparison between various HetNet/MHR placement approaches for the TPB of 200 units. Since MCD approach places only macro eNBs, the power consumed by macro eNB is $100 \%$, whereas for other kind of small cells it is zero. Since ATD and TKD approaches utilize MAHC algorithm for macro eNB placement, the powers consumed by macro eNBs of these approaches are almost the same. In these two approaches, the power consumed by macro eNB is approximately $96.77 \%$. In ATD approach, the remaining $3.23 \%$ power is consumed by the placed micro eNBs. In TKD approach, the small cells like micro- and picocells consume the power of $2.22 \%$ and $1.01 \%$, respectively. The powers consumed by the placed RSs of ACRD, uniform clustering, and fuzzy approaches are almost the same. The power consumed by the small cells of EDMC and SSMPC approaches is $16.45 \%$ and $22.26 \%$, respectively. This verifiably demonstrates that our proposed SSMPC approach effectively utilizes LTE HetNet and MHR to solve the traffic demand and coverage problems experienced by the UEs in the hotspots and dead areas.

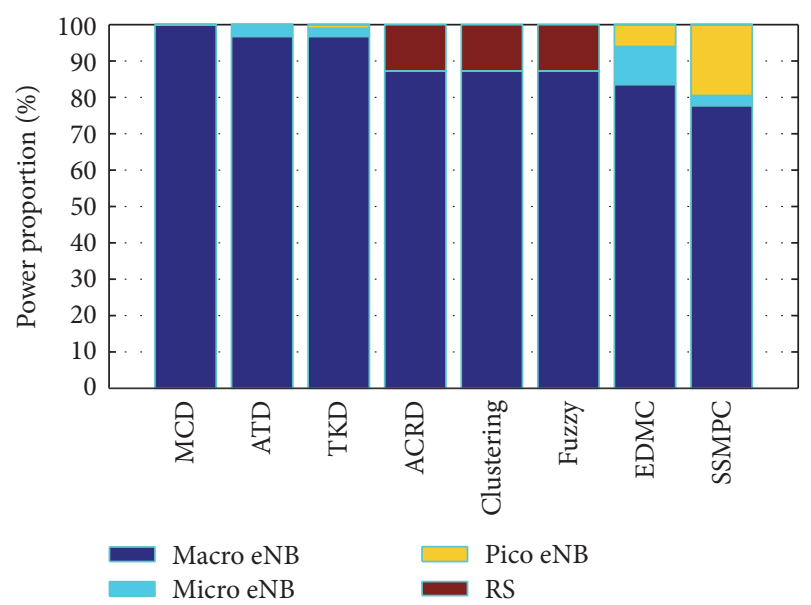

FIGURE 25: Power proportion comparison between different HetNet/MHR placement approaches for the TPB of 200 units.

The SINR of $l$ th UE corresponding to $k$ th macro eNB can be obtained using [33-35]

$$
\operatorname{SINR}_{k, l}=\frac{p_{k, l}^{r}\left|h_{k, l}\right|^{2}}{N_{0}+\sum_{\substack{k^{\prime}=1 \\ k^{\prime} \neq k}}^{E} p_{k^{\prime}, l}^{r}\left|h_{k^{\prime}, l}\right|^{2}+\sum_{m=1}^{B} p_{m, l}^{r}\left|h_{m, l}\right|^{2}},
$$

where $p_{m, l}^{r}$ is the power received by the $l$ th UE from the $m$ th pico eNB and $\left|h_{m, l}\right|^{2}$ is the fading channel gain between the $m$ th pico eNB and $l$ th UE. The SINR of $q$ th UE corresponding to $m$ th pico eNB can be obtained using

$$
\begin{aligned}
& \operatorname{SINR}_{m, q} \\
& =\frac{p_{m, q}^{r}\left|h_{m, q}\right|^{2}}{N_{0}+\sum_{\substack{m^{\prime}=1 \\
m^{\prime} \neq m}}^{B} p_{m^{\prime}, q}^{r}\left|h_{m^{\prime}, q}\right|^{2}+\sum_{k=1}^{E} p_{k, q}^{r}\left|h_{k, q}\right|^{2}},
\end{aligned}
$$

where $p_{m, q}^{r}$ is the power received by the $q$ th UE from the $m$ th pico eNB, $\left|h_{m, q}\right|^{2}$ is the fading channel gain between the $m$ th pico eNB and $q$ th UE, $m^{\prime}$ represents the neighbouring pico eNBs, $p_{m^{\prime}, q}^{r}$ is the power received by the $q$ th UE from the $m^{\prime}$ th pico eNB, $\left|h_{m^{\prime}, q}\right|^{2}$ is the fading channel gain between the $m^{\prime}$ th pico eNB and $q$ th UE, $p_{k, q}^{r}$ is the power received by the $q$ th UE from the $k$ th macro eNB, and $\left|h_{k, q}\right|^{2}$ is the fading channel gain between the $k$ th macro eNB and $q$ th UE.

Figure 26 shows the user experiencing data rate comparison between various HetNet/MHR placement approaches for the TPB of 200 units. Due to the budget constraint, the number of placed serving nodes gets decreased irrespective of the placement approach. This reduces the average per user data rate for all the approaches. This is verifiable by comparing the results in Figures 18 and 26. MCD approach places only macro eNBs. The higher cost nature of macro eNB reduces the number of placed serving nodes. This minimizes the user data rate. Since the other approaches consider the placement of small cells along with the macrocells, the 


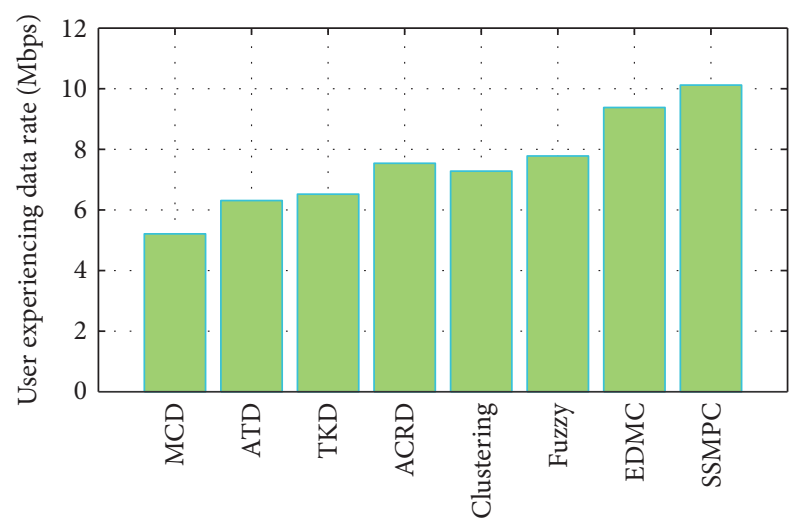

FIGURE 26: User experiencing data rate (Mbps) comparison between different HetNet/MHR placement approaches for the TPB of 200 units.

average per user data rate gets increased than MCD approach. Since the proposed SSMPC approach efficiently chooses the number of serving nodes, their types, and the placement locations, it offers the average per user data rate of $10.12 \mathrm{Mbps}$. The proposed approach increases the data rate approximately by $7 \%$ compared to EDMC approach.

\section{Conclusions}

In this work, four-stage, three-input fuzzy logic based two different approaches are proposed by considering the coverage, cost, power, and interference constraints. The proposed approaches adequately support the decisions on the quantity of serving nodes, their heterogeneity, and the placement sites to fulfill the expected coverage, cost, and traffic demands. The proposed SSMSC approach offers an average SCI of more than 97\%, which is larger than the target SCI of $90 \%$. The PC of SSMSC approach is lesser than the other conventional coverage constraint approaches. The effective SSMSC approach reduces the PC approximately 7\% compared to EDMC approach. The power consumed by the small cells of SSMSC approach is $28.4 \%$ which is $6.2 \%$ more than the conventional EDMC approach. The aggregate power consumed by all the placed serving nodes of SSMSC approach is lesser than the other conventional coverage constraint approaches. To fulfill the target information rate of $1 \mathrm{Mbps}$, the aggregate power required by all the placed serving nodes of SSMSC approach is $154 \mathrm{~W}$ which is much lower than the aggregate power allocated, that is, $232 \mathrm{~W}$. Thus, the proposed approach also fulfills the power constraint.

The SCI performance of our SSMPC approach is better than the other conventional budget constraint approaches. The proposed SSMPC approach offers 3.75\% improvement in SCI over the EDMC approach. The power consumed by the small cells of SSMPC approach is $22.26 \%$ which is $5.81 \%$ more than the conventional EDMC approach. To achieve the target information rate of $1 \mathrm{Mbps}$, the SSMPC approach shows an improvement of approximately $10 \%$ over the EDMC approach. To fulfill the target information rate of $1 \mathrm{Mbps}$, the aggregate power required by all the placed serving nodes of
SSMPC approach is $155.10 \mathrm{~W}$ which is lower than the aggregate power allocated, that is, $180.25 \mathrm{~W}$. Thus, the proposed SSMPC approach also fulfills the power constraint. This verifiably demonstrates that our proposed SSMSC and SSMPC approaches effectively utilize LTE HetNet and MHR to solve the traffic demand and coverage problems experienced by the UEs in the hotspots and dead areas. From Table 4, it is also clear that the proposed approaches are computationally less complex than many of the other conventional approaches. The proposed approaches also minimize the inter-eNB and inter-RS interferences by maintaining sufficient distance between the placed nodes. The inclusion of ICIC and FFR will increase the average user experiencing data rate. The proposed approaches can also be altered by considering the limitation in bandwidth.

The process of cell shrinking and radio resource availability check is automated. This is done periodically. Due to these reasons, the UEs supported by the small cells get increased, which also reduces the network load of macrocells. This also increases the power proportion of the proposed scheme. As a future work, in order to fully exploit the benefits of the self-organizing, the fuzzy system can be replaced by adaptive neuro-fuzzy inference system (ANFIS). The neurofuzzy systems are adaptive, accurate, and learn by themselves.

\section{Competing Interests}

The authors declare that there is no conflict of interests regarding the publication of this paper.

\section{References}

[1] B.-J. Chang, Y.-H. Liang, and S.-S. Su, "Analyses of relay nodes deployment in $4 \mathrm{G}$ wireless mobile multihop relay networks," Wireless Personal Communications, vol. 83, no. 2, pp. 1159-1181, 2015.

[2] A. Khandekar, N. Bhushan, J. Tingfang, and V. Vanghi, "LTEadvanced: heterogeneous networks," in Proceedings of the 2010 European Wireless Conference (EW '10), pp. 978-982, Lucca, Italy, April 2010.

[3] A. Damnjanovic, J. Montojo, Y. Wei et al., "A survey on 3GPP heterogeneous networks," IEEE Wireless Communications, vol. 18, no. 3, pp. 10-21, 2011.

[4] Y.-C. Wang and C.-A. Chuang, "Efficient eNB deployment strategy for heterogeneous cells in 4G LTE systems," Computer Networks, vol. 79, pp. 297-312, 2015.

[5] M. Arthi and P. Arulmozhivarman, "A flexible and cost-effective heterogeneous network deployment scheme for beyond 4G," Arabian Journal for Science and Engineering, vol. 41, no. 12, pp. 5093-5109, 2016.

[6] S. Lee, S. Lee, K. Kim, D. Griffith, and N. Golmie, "Optimal deployment of pico base stations in LTE-Advanced heterogeneous networks," Computer Networks, vol. 72, pp. 127-139, 2014.

[7] I. F. Akyildiz, D. M. Gutierrez-Estevez, R. Balakrishnan, and E. Chavarria-Reyes, "LTE-advanced and the evolution to Beyond 4G (B4G) systems," Physical Communication, vol. 10, pp. 31-60, 2014.

[8] A. B. Saleh, S. Redana, J. Hämäläinen, and B. Raaf, "On the coverage extension and capacity enhancement of inband relay deployments in LTE-advanced networks," Journal of Electrical 
and Computer Engineering, vol. 2010, Article ID 894846, 12 pages, 2010.

[9] J.-Y. Chang and Y.-S. Lin, "A clustering deployment scheme for base stations and relay stations in multi-hop relay networks," Computers and Electrical Engineering, vol. 40, no. 2, pp. 407420, 2014.

[10] Y. Ge, S. Wen, and Y.-H. Ang, "Analysis of optimal relay selection in IEEE 802.16 multihop relay networks," in Proceedings of the IEEE Wireless Communications and Networking Conference (WCNC '09), pp. 1-6, Budapest, Hungary, April 2009.

[11] T. Tsourakis and K. Voudouris, "WiMAX network planning and system's performance evaluation," in Proceedings of the IEEE Wireless Communications and Networking Conference (WCNC '07), pp. 1948-1953, March 2007.

[12] J. Shin, R. Kumar, Y. Shin, and T. F. La Porta, "Multi-hop wireless relay networks of mesh clients," in Proceedings of the IEEE Wireless Communications and Networking Conference (WCNC '08), pp. 2717-2722, April 2008.

[13] I.-K. Fu, W.-H. Sheen, and F.-C. Ren, "Deployment and radio resource reuse in IEEE 802.16j multi-hop relay network in Manhattan-like environment," in Proceedings of the 6th International Conference on Information, Communications and Signal Processing (ICICS '07), pp. 1-5, December 2007.

[14] Y. Yu, S. Murphy, and L. Murphy, "Planning base station and relay station locations in IEEE $802.16 \mathrm{j}$ multi-hop relay networks," in Proceedings of the 5th IEEE Consumer Communications and Networking Conference (CCNC '08), pp. 922-926, IEEE, Las Vegas, Nev, USA, January 2008.

[15] S.-J. Kim, S.-Y. Kim, B.-B. Lee, S.-W. Ryu, H.-W. Lee, and C.-H. Cho, "Multi-hop relay based coverage extension in the IEEE802.16J based mobile WiMAX systems," in Proceedings of the 4th International Conference on Networked Computing and Advanced Information Management (NCM '08), pp. 516-522, Gyeongju, Republic of Korea, September 2008.

[16] H.-C. Lu and W. Liao, "Joint base station and relay station placement for IEEE 802.16j networks," in Proceedings of the IEEE Global Telecommunications Conference (GLOBECOM '09), December 2009.

[17] J.-Y. Chang and Y.-S. Lin, "An efficient base station and relay station placement scheme for multi-hop relay networks," Wireless Personal Communications, vol. 82, no. 3, pp. 1907-1929, 2015.

[18] C.-K. Ting, C.-N. Lee, H.-C. Chang, and J.-S. Wu, "Wireless heterogeneous transmitter placement using multiobjective variable-length genetic algorithm," IEEE Transactions on Systems, Man, and Cybernetics. Part B, Cybernetics, vol. 39, no. 4, pp. 945-958, 2009.

[19] C.-H. Lung and C. Zhou, "Using hierarchical agglomerative clustering in wireless sensor networks: an energy-efficient and flexible approach," Ad Hoc Networks, vol. 8, no. 3, pp. 328-344, 2010.

[20] J. Han, J. Pei, and M. Kamber, Data Mining: Concepts and Techniques, Elsevier, New York, NY, USA, 2011.

[21] Y.-C. Wang, Y.-F. Chen, and Y.-C. Tseng, "Using rotatable and directional $(\mathrm{R} \& \mathrm{D})$ sensors to achieve temporal coverage of objects and its surveillance application," IEEE Transactions on Mobile Computing, vol. 11, no. 8, pp. 1358-1371, 2012.

[22] Fujitsu. High-Capacity Indoor Wireless Solutions: Picocell or Fem-to cell, 2014, http://www.fujitsu.com/downloads/TEL/fnc/ whitepapers/High-Capacity-Indoor-Wireless.pdf.

[23] "3rd Generation Partnership Project; Technical Specification Group Radio Access Network; Evolved Universal Terrestrial
Radio Access (E-UTRA); Relay radio transmission and reception (release 11)," Tech. Rep. TR 36.826, V11.0.0 (2012-09), 2011.

[24] M. Arthi, P. Arulmozhivarman, K. V. Babu, J. J. Joy, and E. M. George, "Technical challenges in mobile multi-hop relay networks," International Journal of Applied Engineering Research, vol. 10, no. 10, pp. 26025-26036, 2015.

[25] B. Bede, "Single input single output fuzzy systems," in Mathematics of Fuzzy Sets and Fuzzy Logic, pp. 105-136, Springer, Berlin, Germany, 2013.

[26] E. Weisstein, Wolfram Mathworld, http://mathworld.wolfram .com/Circle-CircleIntersection.html.

[27] TR 136 942, 'European Telecommunications Standards Institute: LTE; Evolved Universal Terrestrial Radio Access (EUTRA); Radio Frequency (RF) system scenarios (3GPP TR 36.942 version 8.2.0 Release 8)', V8.2.0 (2009-07).

[28] European Telecommunications Standards Institute, "LTE; Evolved Universal Terrestrial Radio Access (E-UTRA); Radio Frequency (RF) requirements for LTE Pico Node B," Tech. Rep. TR 136 931, V9.0.0, 2011.

[29] M. Taranetz, T. Blazek, T. Kropfreiter, M. K. Müller, S. Schwarz, and M. Rupp, "Runtime precoding: enabling multipoint transmission in LTE-advanced system-level simulations," IEEE Access, vol. 3, pp. 725-736, 2015.

[30] D. Martín-Sacristán, J. F. Monserrat, V. Osa, and J. Cabrejas, "LTE-advanced system level simulation platform for IMT-advanced evaluation," Waves, pp. 15-23, 2011, http://www.iteam .upv.es/pdf_articles/42.pdf.

[31] Y. S. Cho, J. Kim, W. Y. Yang, and C. G. Kang, MIMO-OFDM Wireless Communications with MATLAB, John Wiley \& Sons, New York, NY, USA, 2010.

[32] M. Vincent, K. V. Babu, M. Arthi, and P. Arulmozhivarman, "A novel fuzzy based relay node deployment scheme for multi-hop relay network," Procedia Technology, vol. 24, pp. 842-853, 2016.

[33] S. Moon, B. Kim, S. Malik et al., "Cell selection and resource allocation for interference management in a macro-picocell heterogeneous network," Wireless Personal Communications, vol. 83, no. 3, pp. 1887-1901, 2015.

[34] 3GPP, "Comparison of time-domain eICIC solutions," 3GPP R1 104661, 3GPP Std, Madrid, Spain, 2010.

[35] "Summary of the description of candidate eICIC solutions," Tech. Rep. R1-104968, 3GPP Std, Madrid, Spain, 2010. 


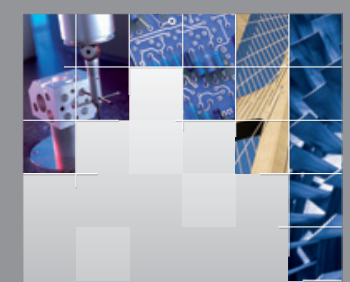

\section{Enfincering}
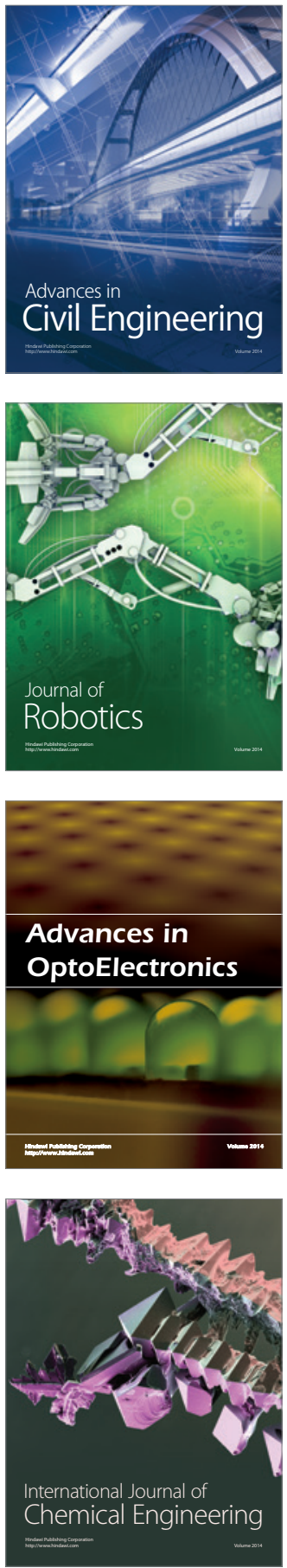

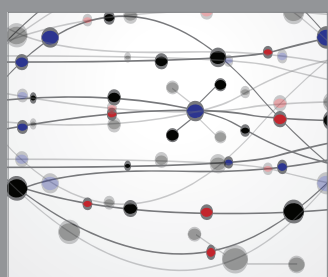

The Scientific World Journal

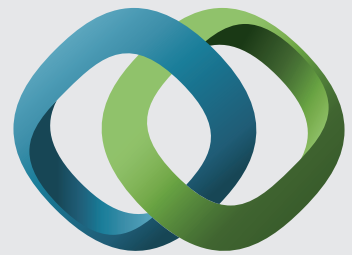

\section{Hindawi}

Submit your manuscripts at

https://www.hindawi.com
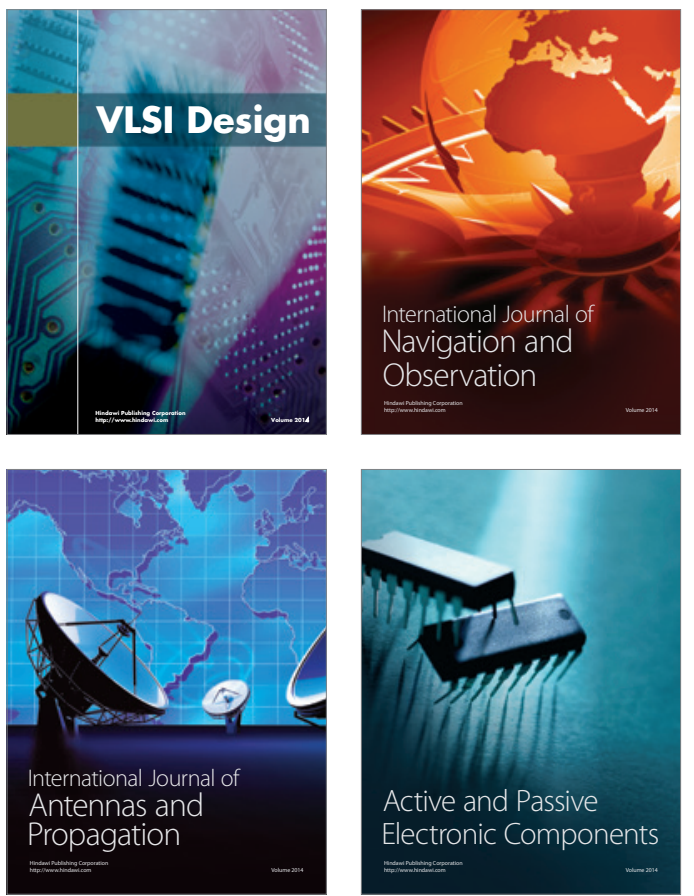
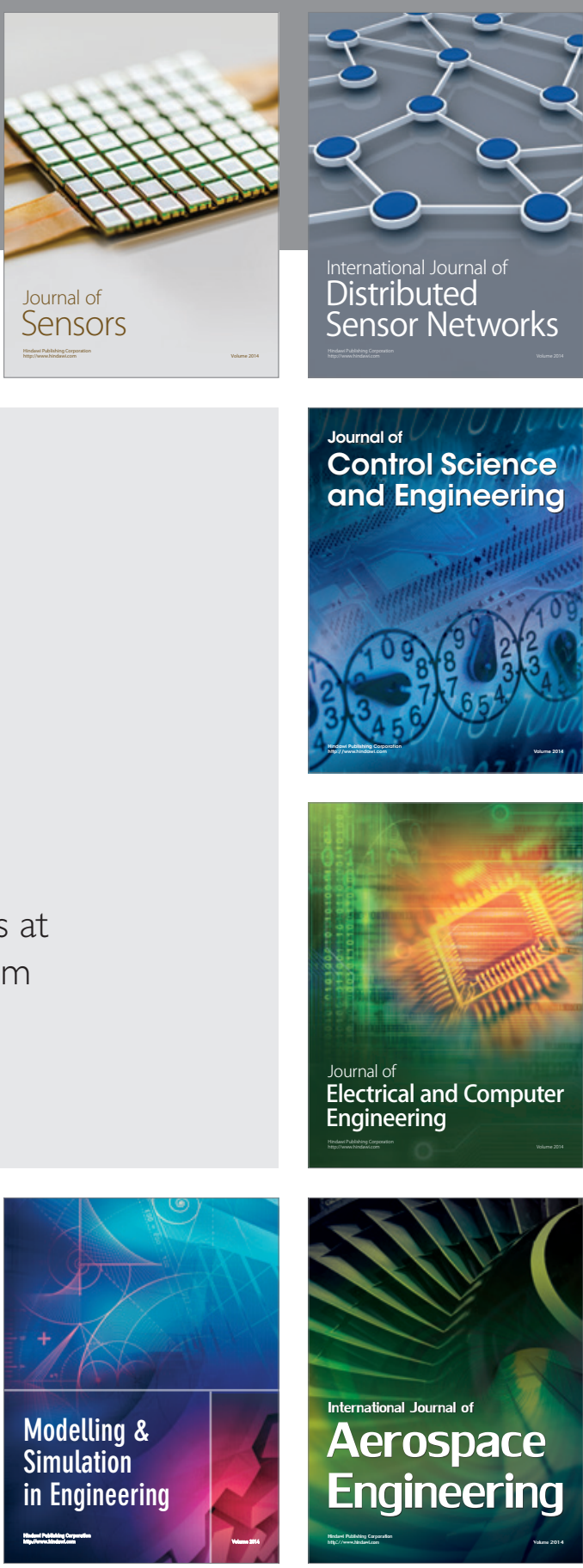

International Journal of

Distributed

Sensor Networks

$-$

Joumal of

Control Science

and Engineering
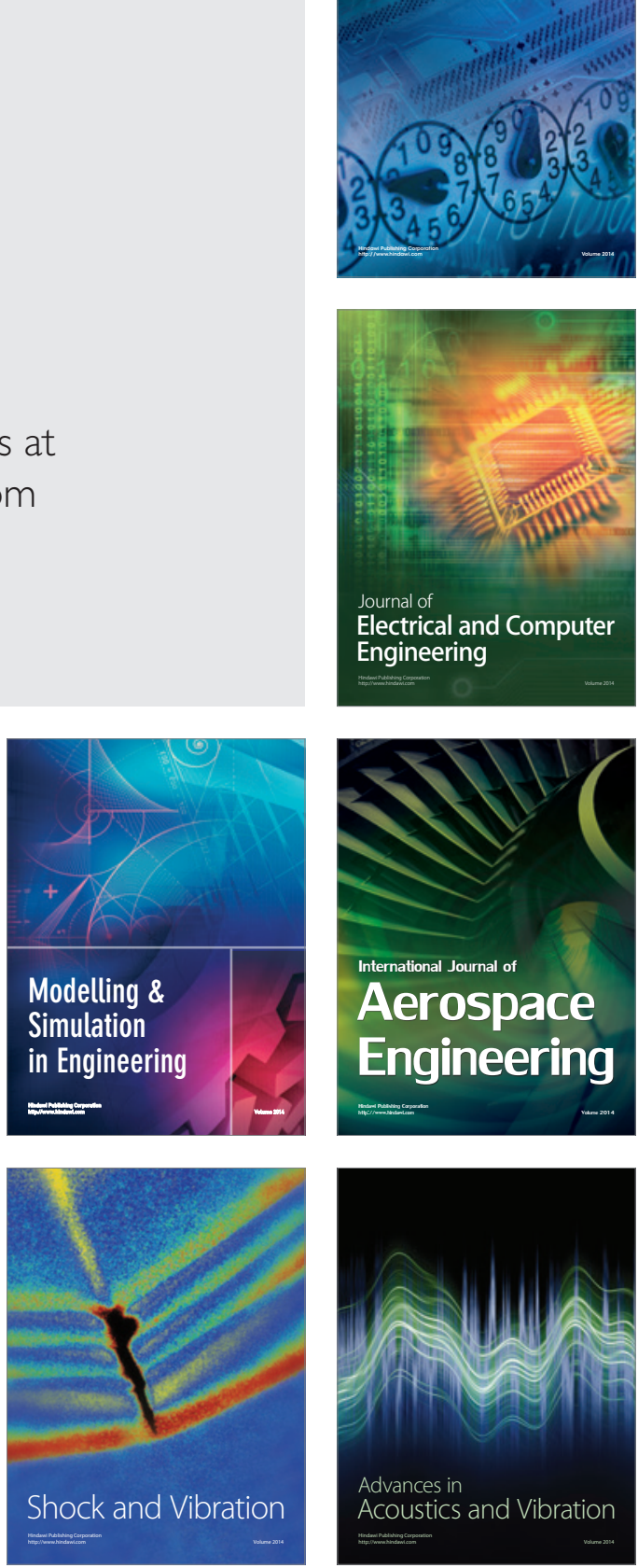